\title{
Experimental study of the aerodynamic noise radiated by cylinders with different cross-sections and yaw angles
}

\author{
E. Latorre Iglesias*, D.J. Thompson, M.G. Smith \\ Institute of Sound and Vibration Research, University of Southampton, Southampton, SO17 1BJ, United Kingdom
}

\begin{abstract}
Vortex shedding from cylinders has been extensively studied due to its occurrence in many engineering fields. Many experimental studies reported in the literature focus on the aerodynamics of the vortex shedding process but the literature about the radiated noise is more scarce. The aim of the work presented here is to extend the available noise data. Aero-acoustic wind tunnel tests were carried out using cylinders with different cross-sections: circular, square, rectangular and elliptical. Flow speeds between 20 and $50 \mathrm{~m} / \mathrm{s}$ were used, corresponding to Reynolds numbers in the range from $1.6 \times 10^{4}$ to $1.2 \times 10^{5}$. The dependence of the noise on the yaw angle, flow speed, cross-sectional shape, angle of attack and radiation angle (directivity) is assessed. The results obtained are compared, where possible, with those found in the literature for similar cases. It is intended that the results can be used for the validation and calibration of numerical and empirical aerodynamic noise prediction models.
\end{abstract}

Keywords:

Noise, cylinder, wind tunnel, aerodynamics, vortex shedding, sound

\section{Introduction}

The phenomenon of vortex shedding from cylinders with different cross-sections has been extensively studied due to its occurrence in many engineering fields. Many experimental works are reported in the literature covering the aerodynamic parameters involved in the vortex shedding process such as Strouhal number and fluctuating lift and drag coefficients. However, the literature about vortex shedding noise is more scarce even though it has applicability to many situations.

Measurements of aerodynamic noise from cylinders obtained in wind tunnels can be used for the validation of Computational Fluid Dynamics (CFD) and Computational AeroAcoustics (CAA) numerical models or for the calibration and validation of semi-empirical and empirical prediction models. However, the available data only cover a limited range of the situations that can be found in real applications. The aim of the current work is to extend the data available to cover additional cases.

Studies were carried out to relate the fluctuating lift force and the noise radiated by a cylinder exposed to a crossflow by Phillips [1], Etkin et al. [2] and Keefe [3] among others. Assuming a sinusoidal fluctuating lift force, they showed that the mean square sound pressure radiated by a smooth circular cylinder in a cross-flow can be expressed in terms of the rms fluctuating lift coefficient $C_{\text {Lrms }}$, the spanwise correlation length $l_{c}$ of the surface pressure fluctuations (normalised by the cylinder diameter, $D$ ) and the Strouhal number $S t$. The Strouhal number is defined as

$$
S t=f_{0} D / U_{\infty}
$$

where $f_{0}$ is the vortex shedding frequency and $U_{\infty}$ is the freestream flow speed. Following this approach, Fujita et al. [4], among others, used the following equation to predict the aerodynamic noise radiated by a cylinder at the vortex

\footnotetext{
*Corresponding author. Tel.:+44 7871658013

Email address: eli1e10@soton. ac.uk (E. Latorre Iglesias)
} 
shedding frequency $f_{0}$

$$
\overline{p^{2}(\mathbf{x})}=\frac{\rho_{0}^{2} U_{\infty}^{6} S t^{2} C_{L r m s}^{2} L l_{c} D}{16 c_{0}^{2} R^{2}} \frac{D_{\mathrm{rad}}(\psi)}{(1-M \cos (\phi))^{4}}
$$

where $\rho_{0}$ is the air density, $c_{0}$ is the speed of sound, $R$ is the distance between the noise source and the observer, which is located at a position $\mathbf{x}$ in the acoustic far-field, $\psi$ is the radiation angle defined as the angle between the axis of the fluctuating lift force (perpendicular to the flow direction) and the observer position, $\phi$ is the angle between the direction of the flow and the observer position, $D_{\mathrm{rad}}(\psi)$ is the directivity function that for a theoretical dipole source is given by $D_{\mathrm{rad}}(\psi)=\cos ^{2}(\psi)$ and $(1-M \cos (\phi))^{4}$ is the convective amplification factor for a dipole source. The Mach number is defined as $M=U_{\infty} / c_{0}$.

Eq. (2) requires that the source is compact, which in this case implies that the cylinder length $L$ should be much smaller than the acoustic wavelength $\lambda$ of the radiated sound, and a far-field condition, which requires that the distance between the cylinder and the observer $R$ should be larger than the acoustic wavelength $\lambda$ and the length of the cylinder $L$ [5]. Moreover, the cylinder is assumed to be rigid and not to vibrate so the normal surface velocity can be assumed to be zero. It is assumed that the Mach number $M$ is small so the quadrupole noise sources, representing the noise produced by the turbulent flow itself, can be neglected [5].

The Strouhal number $S t$, rms fluctuating lift coefficient $C_{L r m s}$ and correlation length $l_{c}$ of a cylinder undergoing vortex shedding are influenced by the Reynolds number $R e=D U_{\infty} / v$, where $v$ is the kinematic viscosity, leading to variations in the noise radiation. The Reynolds number is dependent on the flow speed and cylinder size. Variations of the dimensions of the cylinder may also modify its aspect ratio $(L / D)$. This and the cross-sectional shape affect the flow behaviour around the cylinder. The vortex shedding also depends on the orientation of the cylinder with respect to the incoming flow defined by the yaw angle $\beta$ and angle of attack $\vartheta$, as shown in Figure 1. A yaw angle of $0^{\circ}$ corresponds to a cross flow, whereas at $90^{\circ}$ the cylinder is aligned with the flow.
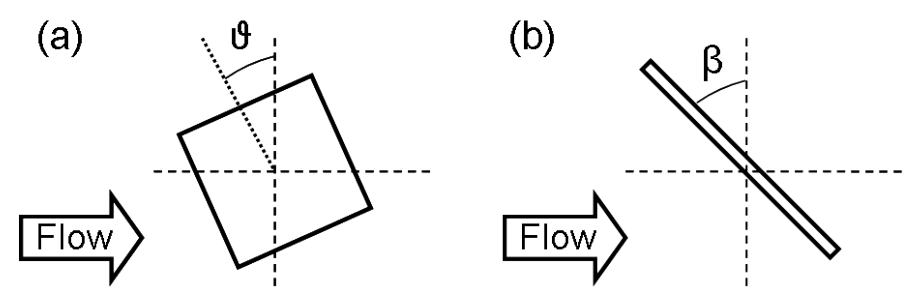

Figure 1: Sketch showing the definition of: (a) the angle of attack $\vartheta$ for a square cylinder (sectional view of the cylinder), (b) the yaw angle $\beta$ (top view of the cylinder).

Many experimental works have been reported on the aerodynamics of cylinders in an airflow. Measurements of the Strouhal number $S t$, fluctuating lift coefficient $C_{\text {Lrms }}$ and spanwise correlation length $l_{c}$ of circular cylinders were carried out by Schewe [6] and by Schlinker et al. [7]. The effect of the cylinder yaw angle on the spanwise correlation length of the surface pressure fluctuations was studied by Hogan and Hall [8] for a yawed circular cylinder. Vickery [9] assessed the influence of the angle of attack and incoming turbulence on the Strouhal number, fluctuating lift coefficient and correlation length of square cylinders. Knisely [10] measured the Strouhal numbers of rectangular cylinders with different slenderness ratios in the range from 0.04 to 4.0 for different angles of attack, while Okajima [11] assessed the effect of the Reynolds number on the Strouhal number of rectangular cylinders with slenderness ratios from 1 to 4 . The effect of the angle of attack on the Strouhal number and fluctuating lift coefficient for elliptical cylinders with different eccentricities was studied experimentally by Modi and Wiland [12] and Modi et al [13].

Experimental studies on the noise radiated by cylinders in such situations are less abundant. King and Pfizenmaier [14] studied the effect of the cylinder aspect ratio on the noise radiated by cylinders with different cross-sections by changing the length of cylinders with one free end for Reynolds numbers in the range $4.4 \times 10^{4}<R e<1.4 \times 10^{5}$. Moreau and Doolan [15] also studied the effect of the aspect ratio of a wall-mounted circular cylinder with a free end on the noise radiation $\left(R e\right.$ from $1.0 \times 10^{4}$ to $1.4 \times 10^{4}$ ), the results being extended to the assessment of the directivity by Porteous et al. [16]. Hutcheson and Brooks [17] studied the effect of incoming turbulence on the noise radiated by circular and square cylinders for Reynolds numbers in the range $3.8 \times 10^{3}$ to $1.0 \times 10^{5}$ using grids of different sizes to generate the turbulence. They assessed the effect of the cylinder surface roughness by placing grit with small particle 
size on the cylinder surface. The directivity of the noise radiated by all the configurations was measured using three microphone positions and the noise from two cylinders in tandem and side-by-side arrangements was also measured.

The effect of the cylinder yaw angle $\beta$ on the vortex shedding noise can be estimated by applying the independence principle [18]. According to this it is assumed that the incident flow speed for an inclined cylinder is the component of the freestream flow speed $\mathbf{U}_{\infty}$ perpendicular to the yawed cylinder centreline $U_{n}=U_{\infty} \cos (\beta)$. Some experimental studies are found on the effect of the yaw angle on the vortex shedding noise from circular cylinders such as those carried out by Yamada et al. for yaw angles up to $45^{\circ}$ [19], by Haramoto et al. up to 50 [20] and by King and Barsikow up to $60^{\circ}[21]$.

The aim of the work presented here is to extend the available experimental databases on the vortex shedding noise radiated by cylinders with different cross-sections in an air flow. Results are presented for the dependence of the noise on the cylinder yaw angle, flow speed, cross-sectional shape (eccentricity and slenderness ratio) and angle of attack (for a square cylinder). Noise directivity is also assessed. A Reynolds numbers range between $1.6 \times 10^{4}$ and $1.2 \times$ $10^{5}$ is used. According to the classification given by Zdravkovich [18] for circular cylinders these Reynolds numbers lie in the subcritical flow regime, in which the boundary layer is laminar and the transition to turbulence occurs in the free shear layer.

The particular motivation of the work reported here is to study the noise radiated by the pantograph of a high-speed train [22], with the tests designed to cover relevant cases that are not available in the literature. For instance, the main struts in a pantograph may be inclined with respect to the incoming air flow with a yaw angle of between $40^{\circ}$ and $75^{\circ}$. In the experiments described here yaw angles between $0^{\circ}$ and $60^{\circ}$ were used for all the cylinder cross-sections, and up to $75^{\circ}$ for the circular cylinder. The eccentricity of the elliptical cylinders and the slenderness ratio of the rectangular cylinders were chosen to be different from those found in the literature in order to increase the number of cases available. For the noise directivity, more measurement points are used than in similar experiments found in the literature and results for yawed cylinders are also included.

The measurements are compared with the independence principle to assess for engineering purposes the effect of the yaw angle on the vortex shedding noise. In addition, where possible, the results obtained are compared with those found in the literature for similar cases. This allows the experimental set-up used in the present experiments to be validated and enables the results available for the calibration of prediction models to be extended to a larger range of cases. More details of these experiments, including the narrow-band spectra measured for each of the test cases, can be found in [23].

The experimental arrangements and analysis approach are described first in section 2. Section 3 presents the results in terms of the yaw angle and compares them with the independence principle, while the effect of flow speed is presented in section 4. Section 5 describes the effect of varying the angle of attack of a square cylinder, section 6 considers the effects of the eccentricity of elliptical cylinders and the slenderness ratio of rectangular ones and, finally, section 7 presents results of the directivity measurements.

\section{Experimental set-up and data analysis}

\subsection{Experimental set-up}

The experiments were carried out in the Institute of Sound and Vibration Research (ISVR) open jet anechoic wind tunnel. This facility provides a high speed flow with low background noise and low turbulence level [24]. The size of the rectangular nozzle was $0.35 \mathrm{~m} \times 0.50 \mathrm{~m}$, which placed limits on the dimensions of the cylinders that could be considered.

Figure 2 shows the cross-section and dimensions of the different cylinders tested. $D$ is the dimension of the cylinder cross-section perpendicular to the flow and $B$ is that parallel to it. The identifier used for each of the cylinders is shown at the bottom left corner: $\mathrm{C}$ stands for the circular cylinder, $\mathrm{S}$ for the square cylinder, $\mathrm{R} 1$ and $\mathrm{R} 2$ for the rectangular cylinders with slenderness ratios $(B / D)$ of 1.33 and 2.0 while $\mathrm{R} 3$ and $\mathrm{R} 4$ stand for the rectangular cylinders with slenderness ratios of 0.75 and 0.5 , obtained by rotating the cylinders R 1 and R2 by $90^{\circ}$. Finally E1 and E2 stand for the elliptical cylinders with eccentricities of 0.63 and 0.75 . For practical reasons, the circular and square cylinders were made of steel and the rectangular and elliptical cylinders were made of wood, all the cylinders having a smooth surface.

For all cases flow speeds of 20, 25, 31.5, 40 and $50 \mathrm{~m} / \mathrm{s}$ were used in order to assess the speed dependence of the noise radiated by the cylinders and to cover a broader Reynolds number range, as shown in Table A.1 (in Appendix 
A). The free stream flow speed was measured using a Pitot tube at the position where the centre of the cylinder was placed, that is $0.7 \mathrm{~m}$ downstream of the centre of the nozzle. Due to the nozzle size, the effective length of the cylinders $(L)$ - defined as the length of the segment of the cylinder placed inside the clean flow - is limited to the values shown in Table A.2. The characteristic dimension $(D)$ is also limited in order to obtain a cylinder aspect ratio large enough to minimize the end effects and to prevent the blockage ratio from becoming too large. This, and the maximum flow speed available in the wind tunnel $(50 \mathrm{~m} / \mathrm{s})$, limited the maximum Reynolds number $R e$ that could be achieved.
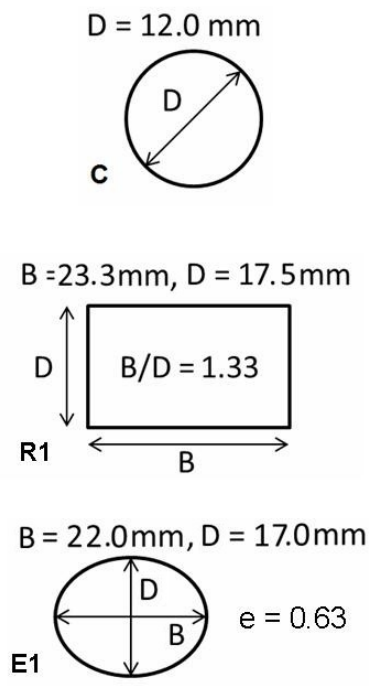
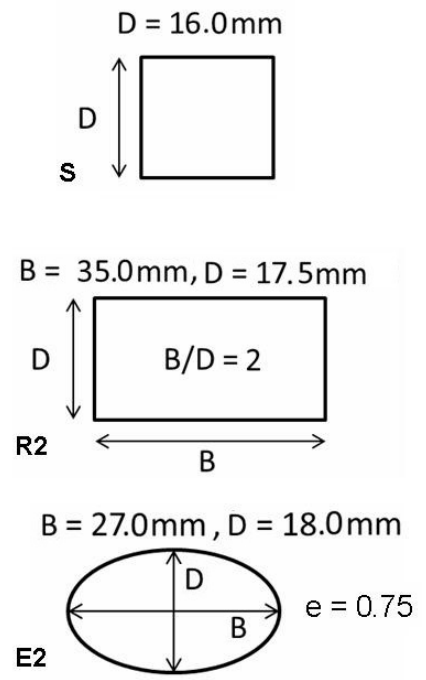

Figure 2: Cross-sections, dimensions and identifier of the cylinders used during the tests.

Yaw angles of $15^{\circ}, 30^{\circ}, 45^{\circ}$ and $60^{\circ}$ were used for all the cylinder configurations and an additional angle of $75^{\circ}$ was evaluated in the case of the circular cylinder. As mentioned above, the vortex shedding noise for a yaw angle of $75^{\circ}$ is of special interest for the application to the aerodynamic noise from a train pantograph as this is the maximum inclination angle for the main and control struts. However, in order to obtain this yaw angle in the tests very long cylinders were required and for practical reasons this was only considered for the circular cylinder.

Angles of attack of $10^{\circ}, 15^{\circ}, 30^{\circ}$ and $45^{\circ}$ were measured for the square cylinder normal to flow (yaw angle of $0^{\circ}$ ). Symmetry was assumed in terms of rotating the square cylinder in the upstream (negative angles of attack) or downstream (positive angles of attack) direction, in accordance with the results shown by Yamada and Fujita [19].

Figure 3 shows the experimental set-up used. The cylinders were attached to two vertical stands, which can be seen in Figure 3(a), that were placed outside the flow to avoid unwanted noise from the interaction between them and the air flow. Both stands were wrapped with sound absorptive foam to minimize reflections of the noise from the cylinder. The floor beneath the cylinder was covered with sound absorptive material for the same reason. It was decided not to use end plates on the cylinders in order to facilitate the measurement of the directivity in the transverse plane and to be able to cover high yaw angles (for yaw angles above $45^{\circ}$ the size of the end plates required would make them quite large and the boundary layer developed at one of the cylinder ends would not be negligible).

An array of eight microphones on a circular arc was placed lengthwise with respect to the air flow direction, as shown in Figure 3(b), in order to measure the directivity of the noise from the cylinders. This configuration allowed radiation angles between $-45^{\circ}$ and $60^{\circ}$ to be covered in steps of $15^{\circ}$. For lower radiation angles the microphone would be shielded by the inlet nozzle while for higher radiation angles the microphone would be placed within the air flow, thereby increasing the background noise.

In order to avoid these drawbacks, and to cover higher radiation angles, a second array of four microphones was positioned transverse to the flow direction, as shown in Figure 3(c). The transverse array covered angles from $45^{\circ}$ to $90^{\circ}$ in steps of $15^{\circ}$. The angle of $0^{\circ}$ in both planes is covered by microphone 4 . In order to avoid the screening effect of the stands when they are positioned for measuring cylinders with a yaw angle of $0^{\circ}$, they were placed $7^{\circ}$ upstream of the cylinder. Symmetry in both the parallel and transverse directions is expected for the directivity of the noise 
radiated by the cylinder. Both arrays covered $45^{\circ}$ and $60^{\circ}$ so, by comparing the results obtained, this symmetry can be verified. It is recognised that the fluctuating drag will produce a dipole noise source with its axis in the flow direction and the peak of the noise spectrum at twice the vortex shedding frequency [25]. However, its amplitude is expected to be around one order of magnitude lower than that of the fluctuating lift noise.
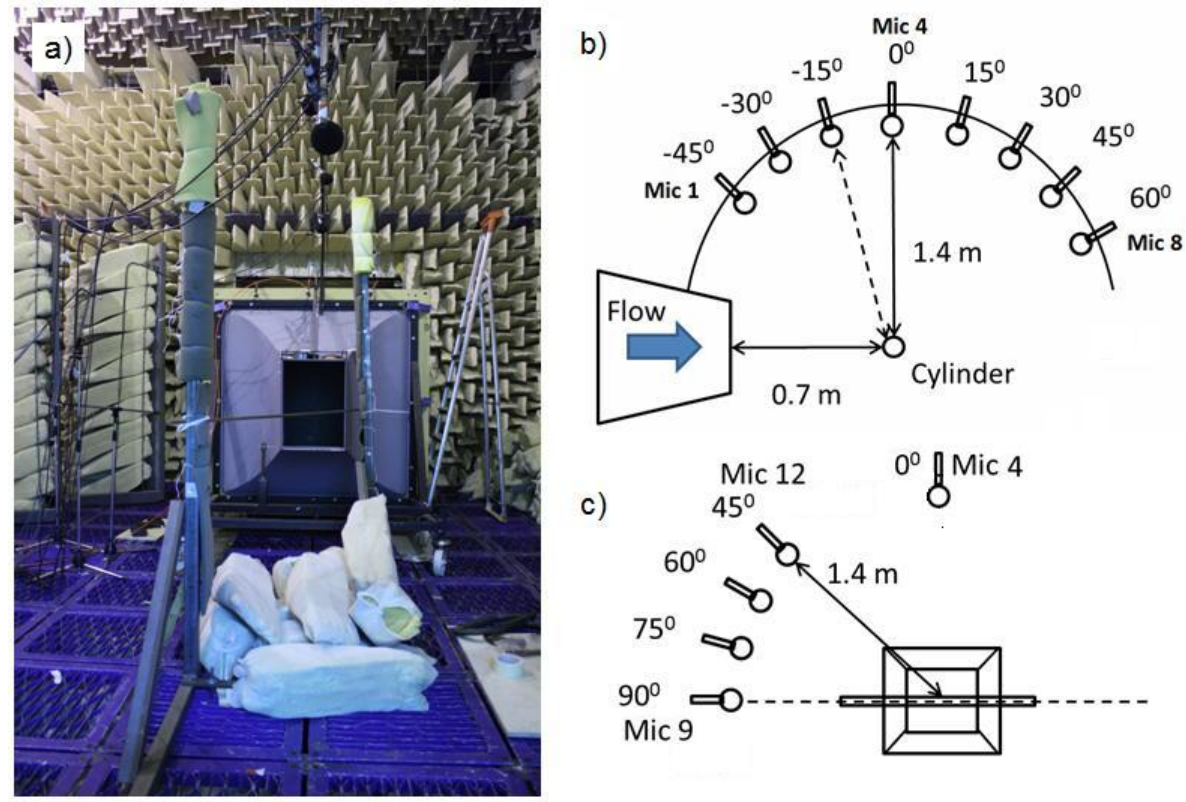

Figure 3: (a) Rear view of the experimental set-up showing the stands holding the square cylinder for the configuration with a yaw angle $\beta=60^{\circ}$. Absorptive foam was placed on the ground to minimize the sound reflections from the floor grids. (b) Side view showing the relative position the longitudinal microphone array and cylinder. (c) Front view showing the transverse microphone array and cylinder. The transverse array was located $7^{\circ}$ upstream of the cylinder to avoid being screened by the stands when they are positioned for measuring cylinders with a yaw angle of $0^{\circ}$.

\subsection{Data analysis}

Microphone 4 was used for all the analysis as its position is perpendicular to the flow direction from the cylinder position so the effects of convective amplification and shear layer refraction [26] can be neglected. The remaining microphones were used only for the directivity measurements.

The microphones were connected to a preamplifier providing the necessary power supply. The output from the preamplifiers was connected to a multichannel acquisition system, which was controlled using a Personal Computer. The whole measurement chain was calibrated for each of the input channels. Time signals of 10 seconds duration for each of the input channels were acquired using a sample frequency of $48 \mathrm{kHz}$. The power spectral density (PSD) was calculated using Welch's method [27] with a Hanning window of 8192 samples and a block overlap of 50\%.

For all the results presented the Strouhal number was calculated from the frequency of the peak of the narrow-band spectrum, with a frequency resolution of $5.8 \mathrm{~Hz}$, which means the Strouhal number is determined with a maximum error of $10 \%$, obtained for the lowest vortex shedding frequency measured (58 Hz for the rectangular cylinder case R3, see Table A.3 in Appendix A), but mostly is found to within 2-3\%.

\subsubsection{Narrow-band noise spectra}

Figure 4 shows the narrow-band PSD spectra of the noise radiated by the circular cylinder for different yaw angles and different flow speeds. As the yaw angle increases both the vortex shedding frequency and the amplitude of the spectral peak decrease. It can been seen how the broadband noise at frequencies above the vortex shedding frequency also decreases with the increasing yaw angle, except for the yaw angle of $75^{\circ}$ for which the broadband noise is dominated by the background noise. Hogan and Hall [8] found that the broadband noise component of the fluctuating wall pressure along the cylinder increases with the yaw angle at frequencies below the vortex shedding 
frequency. However the noise radiated at those frequencies could not be assessed in the present work due to the low signal-to-noise ratio.
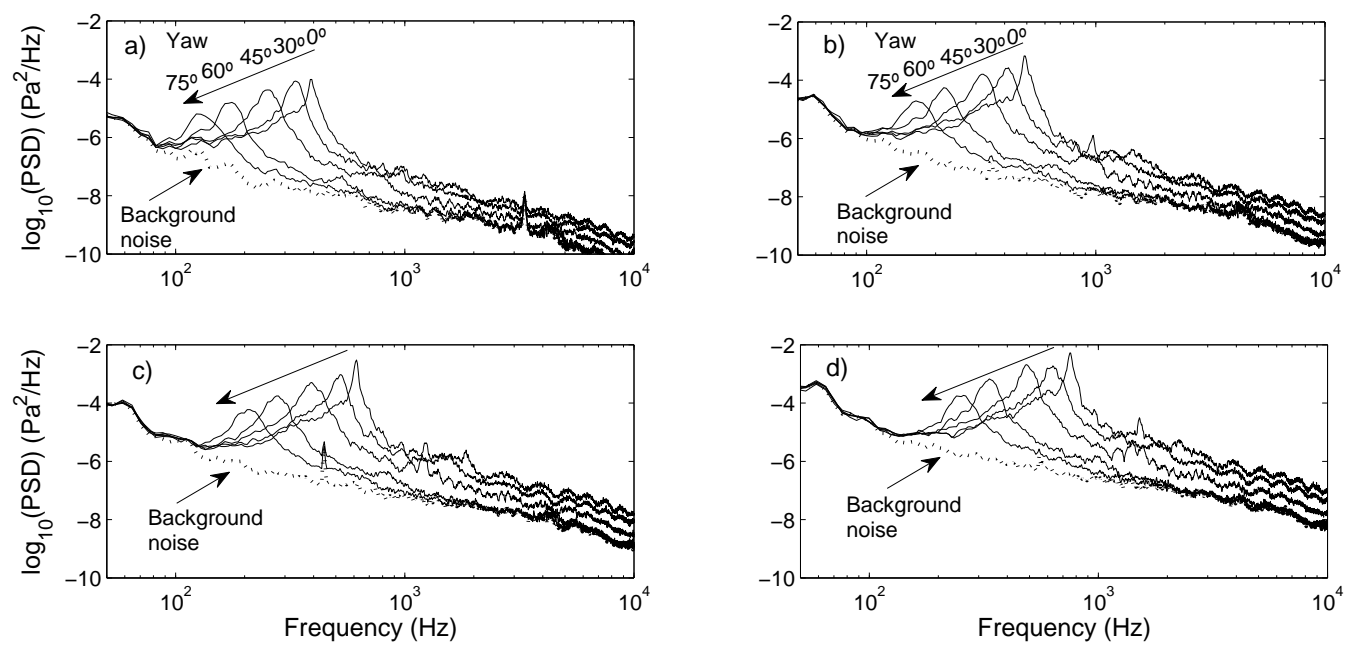

Figure 4: Narrow-band PSD spectra radiated by the circular cylinder for different yaw angles $\beta$ and flow speeds as they were measured by microphone 4 with no corrections applied. (a) $25 \mathrm{~m} / \mathrm{s}$. (b) $31.5 \mathrm{~m} / \mathrm{s}$. (c) $40 \mathrm{~m} / \mathrm{s}$. (d) $50 \mathrm{~m} / \mathrm{s}$.

The narrow-band PSD noise spectra were integrated into 1/3 octave bands and to give an Overall Sound Pressure Level (OASPL) for subsequent analysis. All the results are presented in decibels without any application of the Aweighting. When applicable, the effect of different factors on the results are considered such as: background and shear layer noise, compact source and far field conditions, shear layer and convective amplification and differences in the cylinder length, characteristic dimension and aspect ratio. These are explained in more detail in subsequent sections.

\subsubsection{Background noise and shear layer noise}

The background noise was considered to be the noise when all the elements of the experimental set-up were included except for the cylinder itself. It was measured for all the flow speeds used during the tests.

The absence of end plates makes it important to assess the effect of the jet shear layer impinging on the cylinder because additional noise could be generated. The flow velocity profile was measured using a Pitot tube at a distance of $0.6 \mathrm{~m}$ from the nozzle edge at different crosswise positions through the shear layer. The edge of the shear layer was identified as the position at which the flow speed was approximately $90 \%$ of the mainstream flow speed. Next, the noise was measured when the end of the cylinder was inserted into the flow, with the tip of the cylinder placed at the inner edge of the shear layer. This measurement was used to correct for the shear layer noise. More details about the procedure followed are given in [23].

Figure 5 shows the background noise and shear layer noise measured for the circular cylinder normal to the flow for two different flow speeds: 31.5 and $50 \mathrm{~m} / \mathrm{s}$. Only the shear layer noise of one of the cylinder ends was measured and it was assumed that the overall shear layer noise was $3 \mathrm{~dB}$ higher than this. The measured shear layer noise (SL in Figure 5) was first corrected to remove the background noise. It was then doubled, to allow for the two ends, before adding the background noise again $(\mathrm{BG}+2 \mathrm{SL})$. The result was used to correct the noise measured when a circular cylinder was installed in the air flow with a yaw angle of $0^{\circ}$. It has a strong effect for frequencies below the vortex shedding peak and it also affects some of the frequency bands above the vortex shedding peak. However, it can be seen that no correction is necessary at the frequency of the vortex shedding peak because the peak is much higher than either the background noise or the shear layer noise. The shear layer noise only appears to have an influence on the overall SPL for a yaw angle of $75^{\circ}$. For yaw angles of $60^{\circ}$ or less the difference between the peak noise and the shear layer noise in the same $1 / 3$ octave band is greater than $10 \mathrm{~dB}$ so the effect of the shear layer noise is neglected. For a yaw angle of $75^{\circ}$, the subtraction of the shear layer noise from the measured noise reduced the OASPL by around 2 $\mathrm{dB}$. This correction has been applied to the results. 

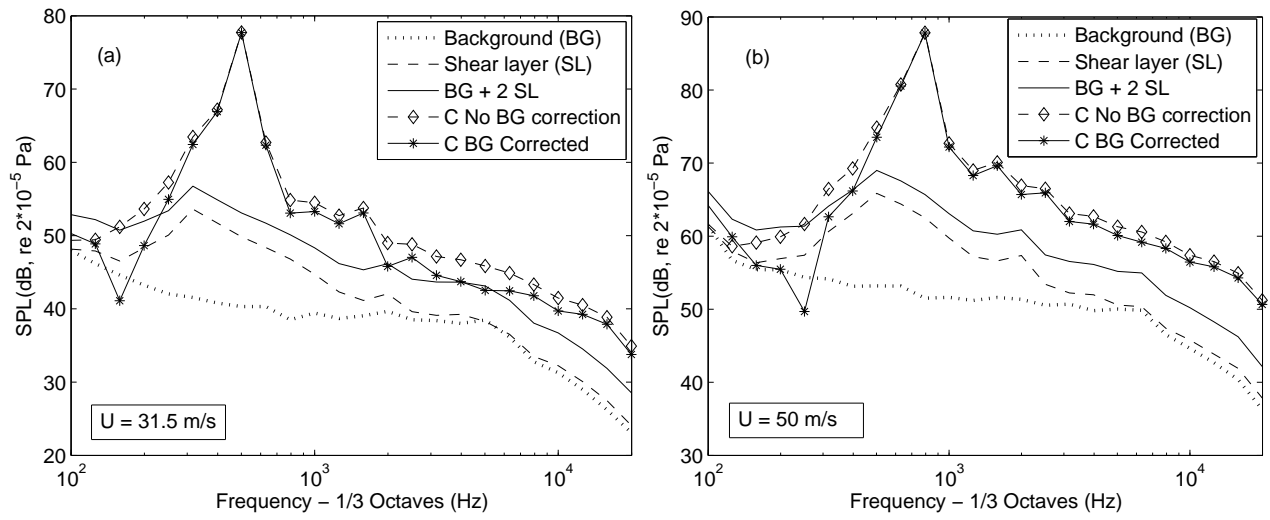

Figure 5: Noise spectra measured for the circular cylinder normal to the flow $\left(\beta=0^{\circ}\right)$. The background noise and shear layer noise are detailed and the effect of the background noise and shear layer noise correction on the noise radiated by the cylinder is shown. (a) Flow speed $31.5 \mathrm{~m} / \mathrm{s}$. (b) Flow speed $50 \mathrm{~m} / \mathrm{s}$.

\subsubsection{Compact source and far field conditions}

The microphone position should ideally satisfy the geometrical and the acoustic far-field conditions. It was found that microphone 4 satisfies the geometrical far-field $(R>2 L)$ condition for all configurations except for the circular cylinder at yaw angle $75^{\circ}$. At the vortex shedding frequency, the acoustic far-field condition $(R>>\lambda)$ is satisfied for slightly less than half the cases. For the square, rectangular and elliptical cylinders at the lowest flow speeds and largest yaw angles this condition is not strictly satisfied. The compact source condition $(L<<\lambda)$ is satisfied for all cases except for the circular cylinder at yaw angle $75^{\circ}$. The maximum and minimum vortex shedding frequencies $f_{0}$ and corresponding acoustic wavelength $\lambda$ as well as the percentage of cases that satisfy each of these conditions are given in Table A.3 in Appendix A for each of the cylinder cross-sections.

In the case of the directivity measurements the same conditions as for microphone 4 are satisfied for the microphones of the longitudinal array, while for the transverse microphone array these are not satisfied because the microphones are close to the cylinder end. This effect has not been taken into account in the present work but it is not expected to be significant. For instance, applying the near-field term proposed by Fujita [19] to the largest wavelength of the vortex shedding peak noise (see Table A.3), which is the most unfavourable case, the correction obtained is 1.6 $\mathrm{dB}$.

\subsubsection{Effect of increasing length of the cylinder with yaw angle}

The length of cylinder inside the flow increased with the yaw angle, which according to Eq. (2) will increase the radiated Sound Pressure Level (SPL). It was corrected for by applying a factor of

$$
\Delta S P L_{\Delta L}=-10 \log _{10}(\Delta L)=10 \log _{10} \cos (\beta)
$$

where $\Delta L$ is the ratio between the cylinder effective length for a yaw angle $\beta$ and the length at yaw angle $0^{\circ}$. The effective length for each of the cylinder cross-sections and yaw angles is given in Table A.4 (in Appendix A).

Because the characteristic dimension $D$ of the cylinders remains constant during the experiments an increase of the length of the cylinder implies an increase of the aspect ratio $L / D$, as shown in Table A.2. For a yaw angle of $0^{\circ}$ the aspect ratio obtained in the present experiments is smaller than 25, which according to King and Pfizenmaier [14] is the minimum value of $L / D$ to consider the end effects negligible. However, the influence of the end effects was not considered in the present results.

\subsubsection{Effect of different characteristic dimension for different cylinder cross-sections}

For practical reasons the characteristic dimension $D$ used during the experiments was slightly different for the different cross-sections, as shown in Figure 2. The implication of this is two-fold: first the Reynolds number obtained is slightly different for the different cross-sections. It is assumed that, for the Reynolds number range covered in the 
present experiments $\left(1.6 \times 10^{4} \leq R e \leq 1.2 \times 10^{5}\right)$, the flow behaviour will be unaffected. Second, the noise amplitude is dependent on the characteristic dimension of the cylinder, as shown in Eq. (2). In order to account for that a second correction factor is applied as follows

$$
\triangle S P L_{\Delta D}=-10 \log _{10}(\Delta D)
$$

where $\Delta D$ is the ratio between the values of $D$ for a particular cylinder cross-section and for the circular cylinder. This correction factor is only applied when the OASPL for different cylinder cross-sections is compared.

\subsubsection{Effect of the variation of the incident mean flow speed away from the jet centreline}

Ideally, if the cylinder is placed in the jet laminar core the incident mean flow speed will be the same at all positions in the jet. In the present experiment, the cylinder is placed $0.7 \mathrm{~m}$ downstream the nozzle. When the cylinder is yawed, one of the ends gets closer to the nozzle and the opposite end is moved further away. Chong et al. [24] measured the transverse distribution of the mean flow speed at different distances from the nozzle, in the ISVR open-jet anechoic wind tunnel for similar Reynolds numbers as those used in the present work.

The speed profiles measured by Chong et al. [24] are used here in order to account for the effect of changes in the incident mean flow speed at different distances from the nozzle and jet centreline. To allow for differences in the dimensions of the rectangular nozzle used by Chong et al. $(0.15 \times 0.45 \mathrm{~m})$ from the present experiments $(0.50 \times 0.35$ $\mathrm{m})$, the distance from the nozzle has been scaled by the hydraulic diameter $D_{h}=(2 H W) /(H+W)$, where $H$ is the nozzle height and $W$ is the nozzle width. For the range of distances from the nozzle covered in the present experiments (maximum $3.0 D_{h}$ when the cylinder is yawed by $75^{\circ}$ ) the incident flow speed along the centreline is assumed to be constant [28].

The yawed cylinder is then divided in nine different sections and, for each of them, the incident flow speed on each is calculated according to its distance from the nozzle and from the jet centreline. It is found that the differences due to this effect are only significant when the cylinder is yawed by $75^{\circ}$; even then the flow speed is unchanged at six sections whereas at the remaining three it is reduced to $0.98 U_{\infty}, 0.91 U_{\infty}$ and $0.86 U_{\infty}$. This variation will lead to some spreading of the vortex shedding peak but, for simplicity, the average flow speed over the cylinder sections $\left(0.97 U_{\infty}\right)$ is used here to represent the incident flow speed of the whole cylinder. The relevant corrections are applied when calculating the Strouhal number and the overall SPL for a circular cylinder yawed by $75^{\circ}$. The resulting change in SPL is $-0.8 \mathrm{~dB}$.

\subsubsection{Effect of the cylinder vibration}

The cylinders used are assumed to be rigid and not to vibrate. However, during the experiments longer cylinders were used for the highest yaw angles making the cylinder more flexible and prone to vibrate, especially for the yaw angle of $75^{\circ}$. Blake [29] showed that, when the vortex shedding frequency is close to the resonance frequency of the cylinder, the vibration may alter the amplitude of the vortex shedding peak and could also affect the Strouhal number obtained. Leehey and Hanson found that the vibration of the cylinder can increase the amplitude of the fluctuating lift coefficient and the correlation length for sufficiently high amplitudes of vibration [30].

In the present experiments the vibration of the cylinder was not measured and its effect on the cylinder vortex shedding was not accounted for. Figure 6 shows the increase of the vortex shedding frequency with the free stream flow speed for different yaw angles of the circular cylinder. The increase is approximately linear for all the yaw angles. If the vortex shedding frequency were close to one of the resonance frequencies of the cylinder then the measured frequency of the noise spectral peak would be expected to be constant for a certain range of flow speeds, instead of having a linear increase. The trends observed in Figure 6 suggest that the cylinder vibration is not affecting the measured vortex shedding frequencies and it will be disregarded.

\subsubsection{Shear layer and convective amplification}

For the directivity measurements the effects of shear layer refraction and sound convection by the mainstream flow were corrected according to the method proposed by Amiet [31]. The effect of convective amplification was removed using the theoretical factor $(1-M \cos (\phi))^{4}$ [32], where $\phi$ is the angle between the observer position and the flow direction, as shown in Eq. (2). The values of the corrections applied can be found in Table A.5 and Table A.6 (in Appendix A). 


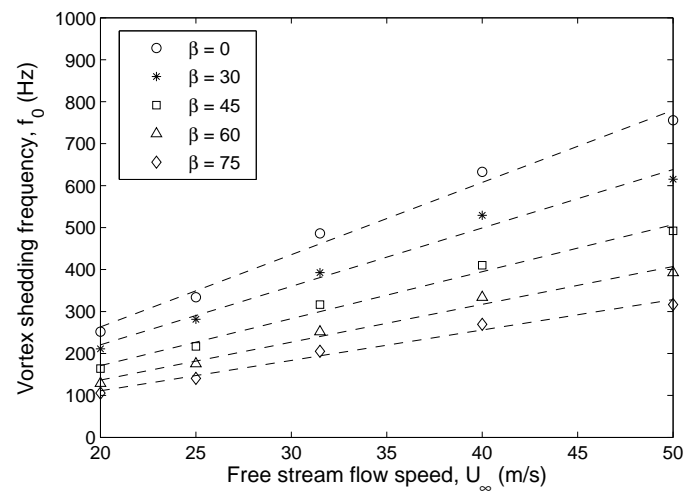

Figure 6: Variation of the vortex shedding frequency of the circular cylinder with the free stream flow speed for different yaw angles.

The sound convection inside the jet and the sound refraction when crossing the shear layer will modify the path followed by the sound rays from the cylinder to each of the microphones and consequently the effective value of the radiation angle $\psi$. Applying Amiet's method for an infinitely thin shear layer [31] a maximum difference of $3^{\circ}$ is obtained (for $50 \mathrm{~m} / \mathrm{s}$, the maximum flow speed used) between the value of $\psi$ with and without considering sound convection and refraction in the shear layer. For simplicity when presenting the results the values of $\psi$ are not corrected.

The thickness of the shear layer increases downstream of the nozzle. Consequently the spanwise flow speed profiles vary depending on the distance from the nozzle, the mean flow speed decreasing when moving away from the nozzle centreline. During the experiments the centre of all the cylinders was kept at the same position for all yaw angles. However, for increasing yaw angles one of the ends of the cylinder gets closer to the nozzle and the opposite one gets further away, the incident flow speed being different along the cylinder. The effect that this can have in the measurements was not accounted for.

\subsubsection{The independence principle}

The independence principle [18] is an approximation that can be used to assess the effect of the yaw angle on the frequency and the amplitude of the vortex shedding noise. Its validity will be assessed through comparisons with the measured results. The incident flow speed $\mathbf{U}_{\infty}$ is decomposed into two perpendicular components, one normal to the yawed cylinder centreline and the other one parallel to it. The normal flow speed component, $U_{n}$, is given by

$$
U_{n}=U_{\infty} \cos (\beta)
$$

For large yaw angles the value of $U_{n}$ is thus much smaller than $U_{\infty}$, e.g. $50 \%$ for $\beta=60^{\circ}$ and $26 \%$ for $\beta=75^{\circ}$.

According to this principle, a cylinder inclined at yaw angle $\beta$ is assumed to be equivalent to a cylinder normal to the flow $\left(\beta=0^{\circ}\right)$ with an incident flow speed $U_{n}$. For inclined cylinders the Strouhal number $S t$, the rms fluctuating lift coefficient $C_{\text {Lrms }}$ and the correlation length $l_{c}$ are all expected to depend on the yaw angle, and therefore the noise radiation is also expected to depend on it. If an equivalent cylinder normal to the flow is used as proposed by the independence principle the dependence of the noise on the yaw angle can be approximated simply by accounting for the variation of the incident flow speed.

Considering $U_{n}$ as the incident flow speed and substituting Eq. (5) in Eq. (1) the dependence of the vortex shedding frequency on the yaw angle is given by

$$
f_{0}(\beta)=f_{0} \cos (\beta)
$$

so according to the independence principle the vortex shedding frequency of a yawed cylinder will change by a factor $\cos (\beta)$. The 'normal Strouhal number' $S t_{n}$ for a yawed cylinder is then defined as

$$
S t_{n}=f_{0}(\beta) D / U_{n}
$$


If Eq. (5) is substituted in Eq. (2) then the dependence of the mean-square sound pressure radiated by a cylinder on the yaw angle can be expressed simplistically as

$$
\overline{p^{2}(\beta, \mathbf{x})}=\overline{p^{2}(0, \mathbf{x})} \cos ^{6}(\beta)
$$

Hence the variation of the SPL with the yaw angle can be obtained by applying a factor $10 \log _{10}\left(\cos ^{6}(\beta)\right)$. This is compared with the measured results in section 3 .

\section{Dependence on the yaw angle}

In this section the effect of the yaw angle on the Strouhal number and amplitude of the vortex shedding noise is assessed using the results obtained during the present wind tunnel tests. These results are compared with those expected according to the independence principle and with results found in the literature.

In addition the bandwidth $B_{f}$ of the vortex shedding peak is considered as this is found to vary with the yaw angle. It is defined here as the frequency range between points that are $10 \mathrm{~dB}$ below the spectral peak, obtained from the narrow-band noise spectra. The results are expressed in terms of the relative bandwidth which is defined as $B_{f} / f_{0}$, where $f_{0}$ is the vortex shedding frequency.

\subsection{Circular cylinder}

According to the independence principle the Strouhal number should vary with the yaw angle according to a factor $\cos (\beta)$, so that $S t_{n}$ should be independent of the cylinder yaw angle. For the circular cylinder, Figure 7(a) shows the variation of the normal Strouhal number $S t_{n}$ with the yaw angle. Results are shown for the five flow speeds tested as well as the average. The average values of $S t_{n}$ are nearly constant with the yaw angle for angles up to $45^{\circ}$, with a slight increase for $60^{\circ}$ and significant increase for $75^{\circ}$. There is a significant variability of the results with the flow speed, with variations of around $20 \%$ in some cases. Figure 7(b) shows the variation of the normal Strouhal number with the Reynolds number. The measured values for the lower Reynolds numbers, which correspond to the flow speeds of 20 and $25 \mathrm{~m} / \mathrm{s}$, are significantly lower than those measured for the higher Reynolds numbers.

The results in Figure 7(a) are compared with those obtained by Yamada et al. [19], by King and Barsikow [21] and by Haramoto et al. [20] who measured the vortex shedding noise from circular cylinders for a similar Reynolds number range as that covered in the present investigations (see Table A.1 and Table A.7 in Appendix A). The trend followed by the measured $S t_{n}$ with yaw angle is in good agreement with those measured by Yamada et al. and King and Barsikow. The measured average value is slightly lower than that measured by those authors, although this is mainly affected by the results at low flow speeds; for the flow speeds of $31.5,40$ and $50 \mathrm{~m} / \mathrm{s}$ the present results are close to those in the literature. The results measured by Haramoto et al. are also nearly constant with the yaw angle but the values of $S t_{n}$ are higher than those from the present work and the other authors.

The reason that the present results are lower than those found in the literature could be that the aspect ratio $L / D$ used in the current experiments is not high enough to allow the end effects to be neglected. Gowda found that the threedimensional flow produced by the cylinder free ends affected the Strouhal number of the vortex shedding produced by a circular cylinder in an air flow [33]. Gowda found that an aspect ratio $L / D$ above 45 is necessary to obtain the same Strouhal number as those measured using end-plates. However, King and Pfizenmaier found that, with an aspect ratio $L / D$ of 25 , the Strouhal number obtained for a circular cylinder with a single free end in an air flow was the same as those measured with end plates [14]. In the present experiments $L / D$ is 22 for $0^{\circ}$, increasing to 51 at $60^{\circ}$, see Table A.2. However, it is found that the normal Strouhal number does not increase when the cylinder is yawed, even though the aspect ratio increases substantially, except for a yaw angle of $75^{\circ}$. Therefore it does not appear that the slightly lower values of the measured normal Strouhal number compared with the values found in the literature can be attributed to the limited aspect ratio.

It has been seen that the normal Strouhal number is significantly higher for a yaw angle of $75^{\circ}$. This increase could be a consequence of changes in the flow behaviour around the cylinder but this was not proven. Although the vibration of the cylinder could also affect the Strouhal number obtained [29], it has already been shown in Figure 6 that it is unlikely to have had an influence.

Figure 8(a) shows the OASPL plotted against yaw angle. These are shown normalised to the case of the circular cylinder normal to the flow. The length correction shown in Eq. (3) has been applied. The results have been averaged 

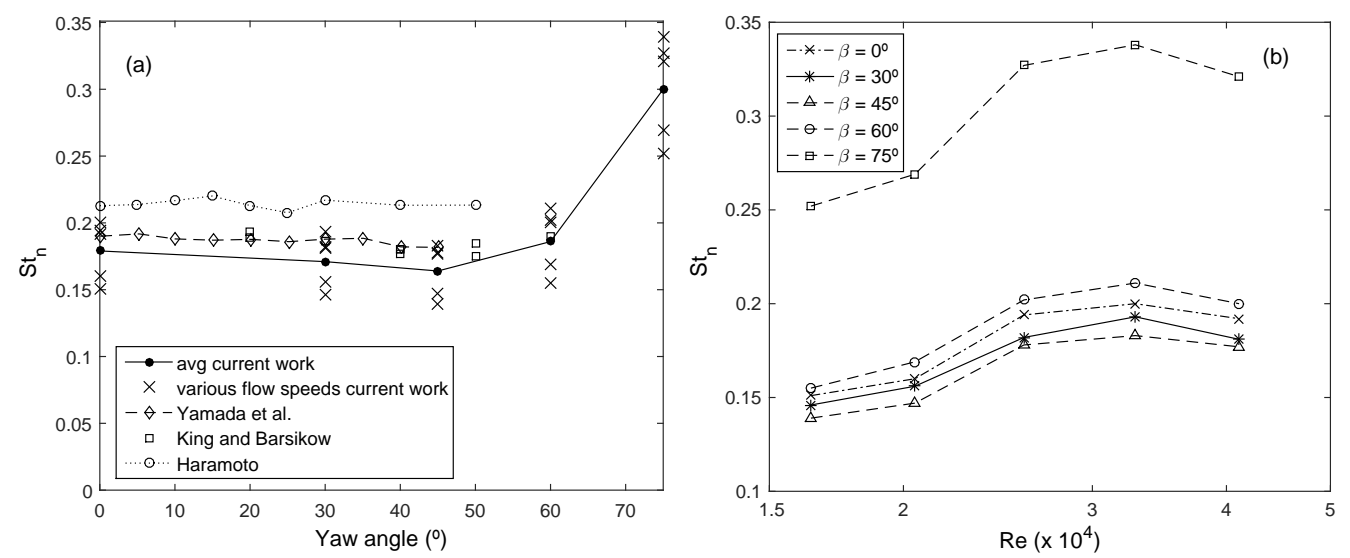

Figure 7: Circular cylinder. (a) Variation of the normal Strouhal number $S t_{n}$ with the Reynolds number. The results are compared with those those measured by Yamada et al. [19], by Haramoto et al. [20] and by King and Barsikow [21]. (b) Variation of the normal Strouhal number $S t_{n}$ with the yaw angle.
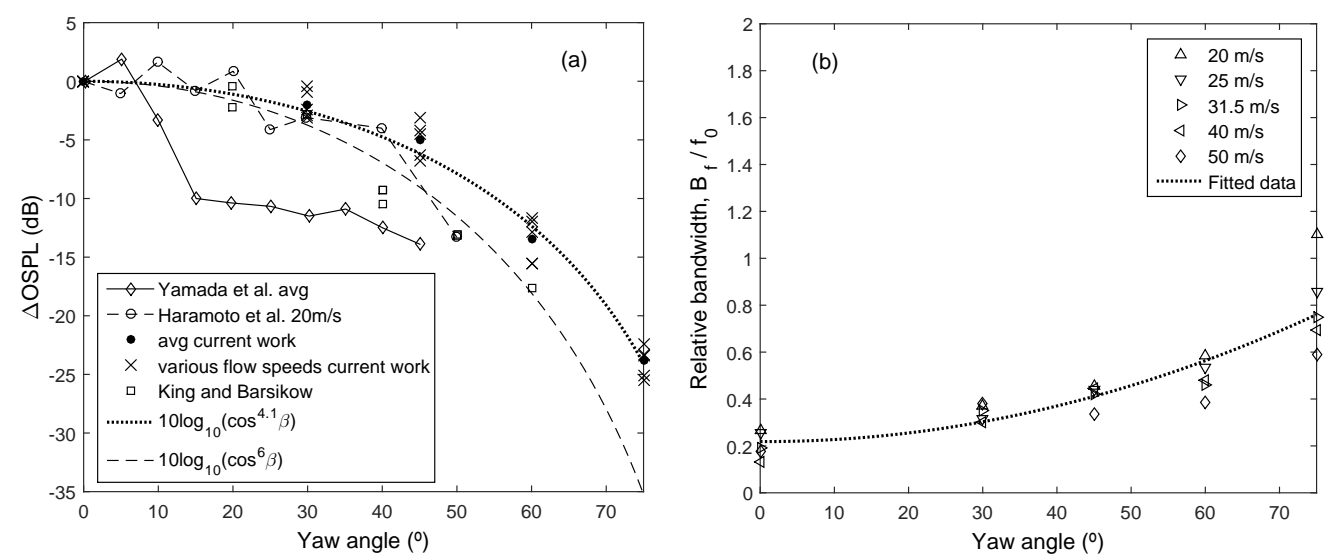

Figure 8: (a) Variation with the yaw angle $\beta$ of the difference between the OASPL radiated by a circular cylinder normal to the flow and radiated by the same circular cylinder yawed by different angles. The length correction shown in Eq. (3) was applied. The results are compared with those obtained by using the theoretical factors $60 \log _{10}(\cos (\beta))$ and $37 \log _{10}(\cos (\beta))$ and with those measured by Yamada et al. [19], Haramoto et al. [20] and King and Barsikow [21]. (b) Variation of the relative bandwidth with the yaw angle for a circular cylinder.

for the different flow speeds and they are compared with the factor $10 \log _{10}(\cos (\beta))^{\gamma}$ for two different values of $\gamma$ : $\gamma=6$ as proposed for the independence principle (Eq. (8)) and $\gamma=3.7$. The latter value was found by applying a least squares fit to the average results.

The exponent $\gamma=6$ does not provide such a good agreement with the measurements. However, the results of King and Barsikow [21] are closer to the trend obtained using $\gamma=6$. The results are also compared with those obtained by Yamada et al. [19] and Haramoto et al. [20] for an inclined circular cylinder after being corrected by cylinder length by applying Eq. (3). The results from Haramoto et al. seem to follow $\gamma=6$ but with an error of up to $3 \mathrm{~dB}$ for some of the yaw angles. In the results obtained by Yamada et al. there is an abrupt decrease of the OASPL for yaw angles between $5^{\circ}$ and $15^{\circ}$, which was not obtained in the other results.

According to the independence principle the SPL should reduce more rapidly for higher yaw angles. This is true of the present results up to a yaw angle of $60^{\circ}$. However, for a yaw angle of $75^{\circ}$ the decrease is smaller than expected, as seen in Figure 8(a). When the cylinder is yawed by $75^{\circ}$ the far-field and compact source approximations are not fulfilled. This may affect the amplitude of the measured noise. However, these effects are expected not to be significant, as discussed in Section 2.2.3 and would in any case suggest that the measured results are underestimated. 
The relative bandwidth of the vortex shedding peak is shown in Figure 8(b) for the circular cylinder. The results for different flow speeds are shown together with the curve obtained by fitting a quadratic function to the average results (for the five flow speeds). The relative bandwidth increases with the yaw angle, especially for the yaw angles of $60^{\circ}$ and $75^{\circ}$. The variability of the results with the flow speed is also higher for these angles. According to the results the relative bandwidth is also speed-dependent, decreasing when the flow speed increases.

\subsection{Square cylinder}

For the square cylinder the normal Strouhal number $S t_{n}$ is plotted against the yaw angle $\beta$ in Figure 9. A nearly constant value of the normal Strouhal number is obtained as the yaw angle is increased. The same trend is found in the results obtained by King and Barsikow [21] for a slightly higher Reynolds number, although their values of $S t_{n}$ are slightly lower than those measured in the present experiments. The variability with the flow speed is small, the maximum variability (less than $15 \%$ ) being found for a yaw angle of $60^{\circ}$.

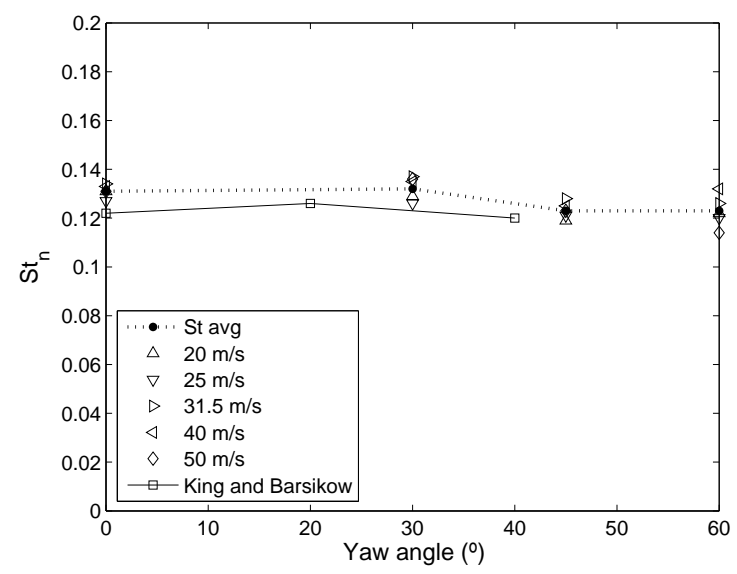

Figure 9: Variation of the normal Strouhal number $S t_{n}$ with the yaw angle $\beta$ for square cylinders. The results are compared with the results obtained by King and Barsikow [21].
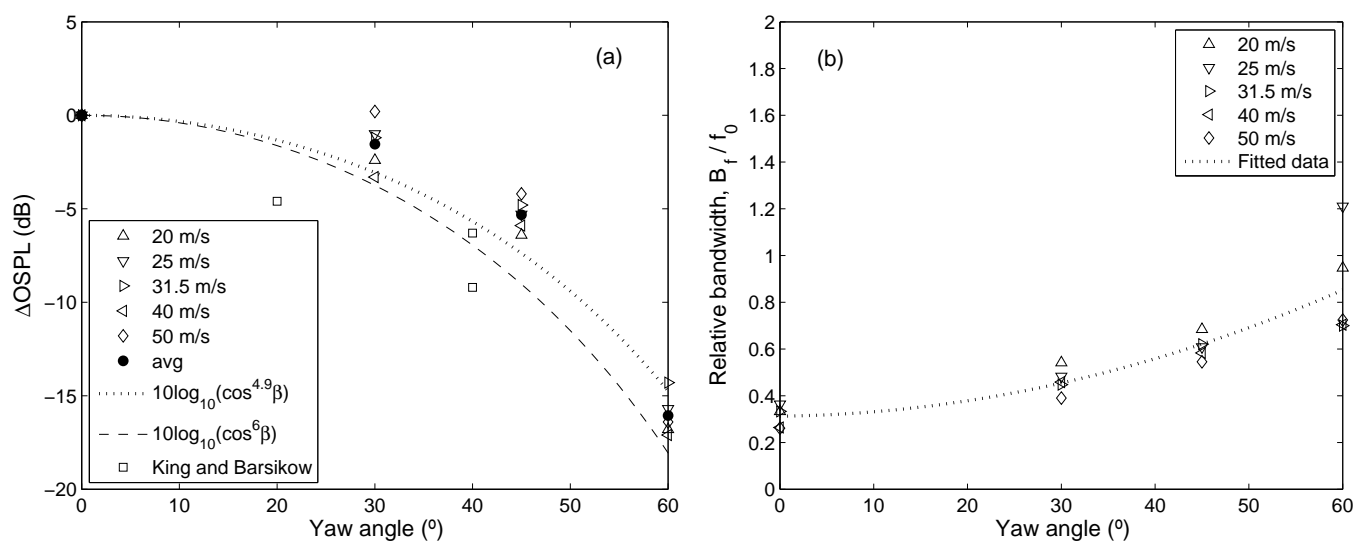

Figure 10: (a) Variation of the difference between the OASPL radiated by a square cylinder normal to the flow and with a yaw angle $\beta$. Correction for the different cylinder lengths was applied according to Eq. (3). The results are compared with the theoretical factors $60 \log _{10}(\cos (\beta))$ and $49 \log _{10}(\cos (\beta))$ and with the results measured by King and Barsikow [21]. (b) Variation of the relative bandwidth with the yaw angle for a square cylinder. 
The OASPL radiated by a square cylinder yawed by different angles $\beta$ is shown normalised to the result for $\beta=0$ in Figure 10(a). The results have been corrected for the different cylinder lengths using the factor shown in Eq. (3). For a yaw angle of $60^{\circ}$ the trend followed is in good agreement with the factor $10 \log _{10}\left(\cos ^{\gamma}(\beta)\right)$, with $\gamma=6$, but the difference is significant for yaw angles of $30^{\circ}$ and $45^{\circ}$. The value of the exponent $\gamma=4.9$ was found from a least squares fit to the average OASPL difference. The results of King and Barsikow [21] show a greater reduction of OASPL with the yaw angle than that found in the present work.

Figure 10(b) shows an increase of the relative bandwidth of the vortex shedding peak with the yaw angle. These results are in good agreement with those from Knisely [10]. He found the bandwidth $B_{f}$ of the peak of the fluctuating lift spectrum to be constant with the flow speed, resulting in a decrease of the relative bandwidth with increasing flow speed. The variability with the flow speed is lower than for the case of the circular cylinder but, nevertheless, slightly higher values of relative bandwidth are obtained for the lower flow speeds, especially for the yaw angle of $60^{\circ}$.

\subsection{Rectangular cylinder}

Figure 11 shows the variation of the normal Strouhal number $S t_{n}$ with increasing yaw angle $\beta$ for the rectangular cylinders R1 $(B / D=1.33)$ and R3 $(B / D=0.75)$. For the cylinder R1 the values of $S t_{n}$ reduce slightly with the yaw angle; the variability with the flow speed is low except for a yaw angle of $60^{\circ}$. However for the cylinder R3 the normal Strouhal number increases slightly with the yaw angle. Cylinders R2 and R4 are not included here as they were only tested for a yaw angle of zero.

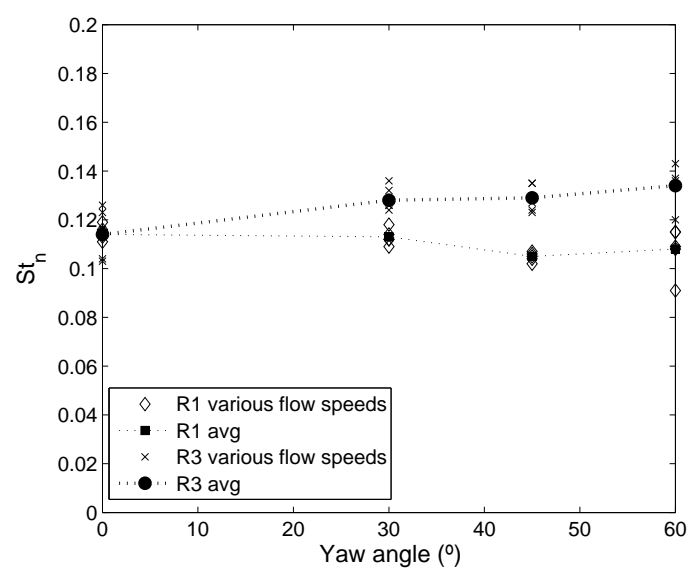

Figure 11: Variation of the normal Strouhal number $S t_{n}$ with the yaw angle $\beta$ for the rectangular cylinders R1 and R3.

Figure 12(a) shows the change in OASPL with yaw angle for cylinder R1. The decrease in level is greater than expected according to the independence principle, especially for $60^{\circ}$. A factor $10 \log _{10}\left(\cos ^{\gamma}(\beta)\right)$ with $\gamma=7.2$ was obtained by a least squares fit. However, a smaller factor appears to fit the results for $30^{\circ}$ and $45^{\circ}$. Figure 12 (b) shows the relative bandwidth. This increases slightly for yaw angles of $30^{\circ}$ and $45^{\circ}$, the increase becoming much steeper for an angle of $60^{\circ}$. The trend followed for $30^{\circ}$ and $45^{\circ}$ is in good agreement with the results obtained for circular and square cylinders, as shown in Figure 8(b) and Figure 10(b). The results for the angle of $60^{\circ}$ are influenced by the background noise, this being the reason for obtaining a large bandwidth.

The vortex shedding peak was not clearly distinguishable in the case of the rectangular cylinder R3 with a yaw angle of $0^{\circ}$, the reason for that being possibly due to a small cylinder aspect ratio. The vortex shedding frequency could be recognized but the amplitude and width of the vortex shedding peak were found to be unreliable. Therefore only the results obtained for yaw angles of $30^{\circ}, 45^{\circ}$ and $60^{\circ}$ are shown in Figure $12(\mathrm{c})$, these being normalised to the results at $30^{\circ}$. A least squares fit was applied to the average data, obtaining a value $\gamma=3.9$. For the rectangular cylinder R3, Figure 12(d) shows a slight increase of the relative bandwidth of the vortex shedding peak with the yaw angle $\beta$, the variability with the flow speed being higher for a yaw angle of $60^{\circ}$. 

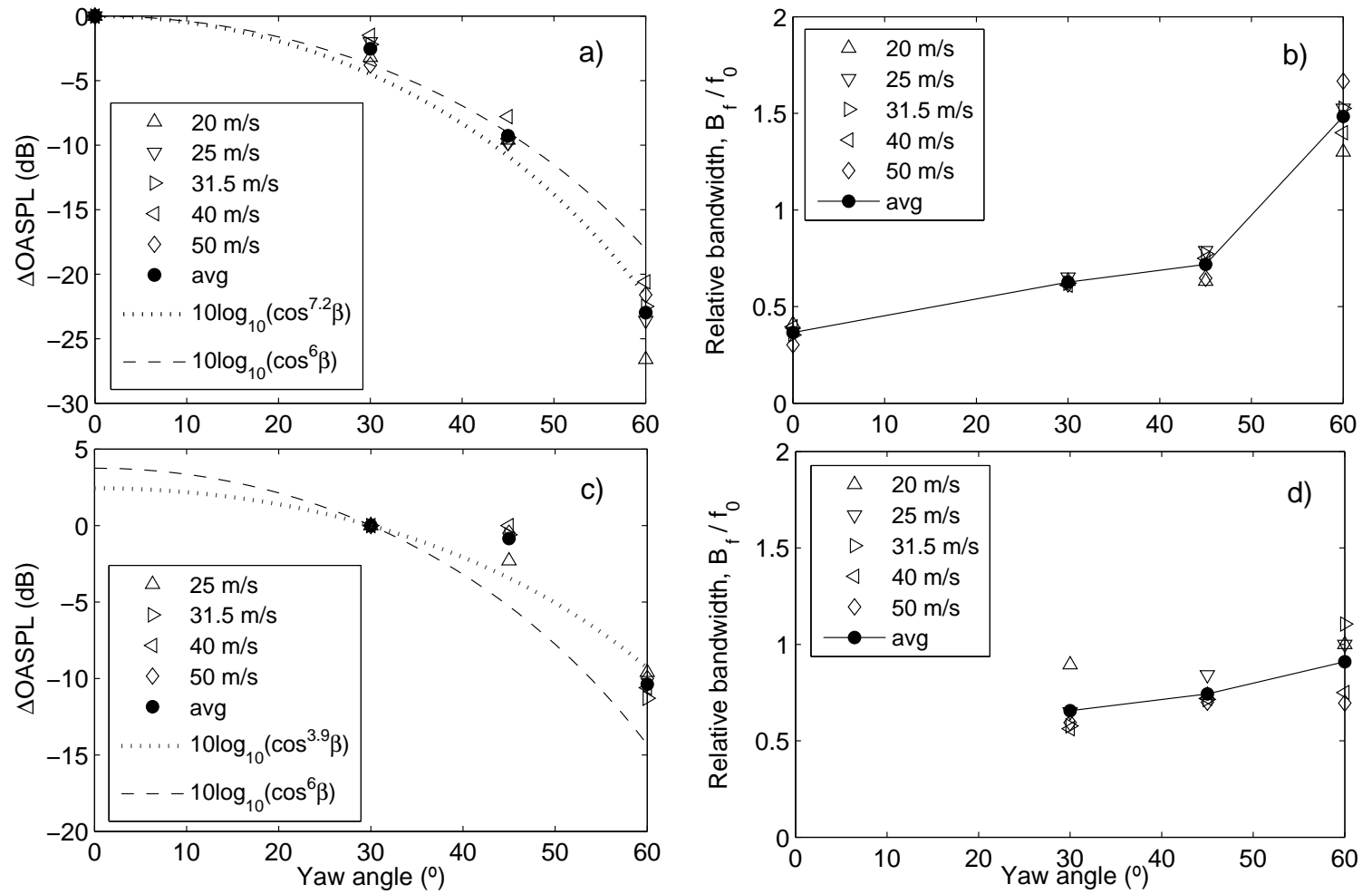

Figure 12: Variation of the OASPL and relative bandwidth with the yaw angle for the rectangular cylinders. A correction for the different cylinder lengths was applied according to Eq. (3). The results are compared with the factor $10 \log _{10}\left(\cos ^{\gamma}(\beta)\right)$, with $\gamma=6$ as proposed by the independence principle and the value of $\gamma$ as obtained by least squares. (a) OASPL vs. yaw angle for the rectangular cylinder R1. (b) Bandwidth vs. yaw angle for the rectangular cylinder R1. (c) OASPL vs. yaw angle for the rectangular cylinder R3. (d) Bandwidth vs. yaw angle for the rectangular cylinder R3.

\subsection{Elliptical cylinder}

For the elliptical cylinders E1 and E2 the normal Strouhal number $S t_{n}$ is nearly constant with the flow speed, as shown in Figure 13. The values of $S t_{n}$ obtained for cylinder E2 are slightly higher than those for cylinder E1. For the cylinder E2 with a yaw angle of $0^{\circ}$ and a flow speed of $50 \mathrm{~m} / \mathrm{s}$ the value of the normal Strouhal number is significantly higher (0.36). This value was not included in the calculation of the average $S t$. In this case, the vortex shedding peak is significantly broader than the others as well as the frequency of the spectral peak increasing.

As for the case of the rectangular cylinder R3, the vortex shedding peak for both elliptical cylinders E1 and E2 was very broad for a yaw angle of $0^{\circ}$ and the OASPL was significantly lower than for larger yaw angles. These results are therefore not included in Figure 14.

For cylinder E1 Figure 14(a) shows that, just taking into account the results for the yaw angles of $30^{\circ}, 45^{\circ}$ and $60^{\circ}$, the value of the exponent $\gamma$ that better fits the results is 5.5. The variability of the results with the flow speed is significant. Unlike the results for the circular, square and rectangular cylinders, the relative bandwidth does not increase with the yaw angle but larger relative bandwidths are obtained for the higher flow speeds, as shown in Figure 14(b).

Similar results are obtained for cylinder E2. Figure 14(c) shows that an exponent $\gamma=5.1$ is obtained after applying a least squares fit to the speed averaged results for yaw angles of $30^{\circ}, 45^{\circ}$ and $60^{\circ}$. The relative bandwidth is again nearly constant with the yaw angle $\beta$ except for a slight decrease for a yaw angle of $30^{\circ}$, as shown in Figure $14(\mathrm{~d})$. However, the variability of the values of the relative bandwidth with the flow speed is large.

\subsection{Comparison of the results with the independence principle}

The feasibility of using the simplifications proposed by the independence principle for the noise amplitude and vortex shedding frequency of yawed cylinders is summarised in this section. Where possible, the error obtained by 


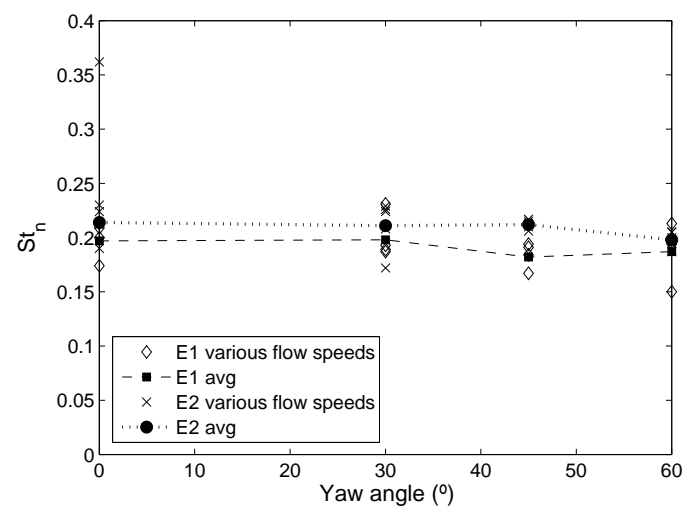

Figure 13: Variation of the normal Strouhal number $S t_{n}$ with the yaw angle $\beta$ for the elliptical cylinders E1 and E2.
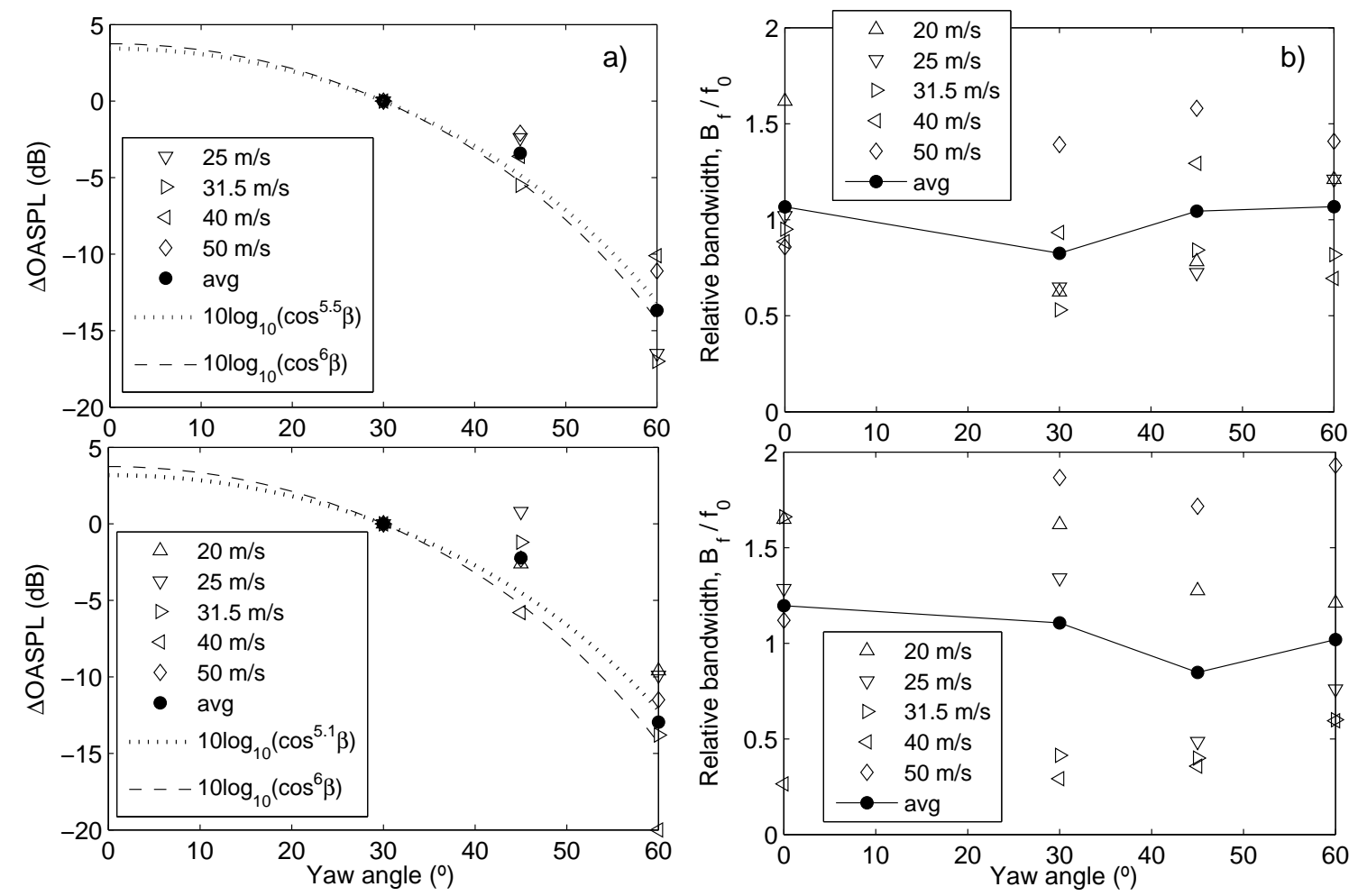

Figure 14: Variation of the OASPL and relative bandwidth with the yaw angle for the elliptical cylinders. A correction for the different cylinder lengths was applied according to Eq. (3). The results are compared with the factor $10 \log _{10}\left(\cos ^{\gamma}(\beta)\right)$, with $\gamma=6$ as proposed by the independence principle and the value of $\gamma$ as obtained by least squares. (a) OASPL vs. yaw angle for the elliptical cylinder E1. (b) Bandwidth vs. yaw angle for the elliptical cylinder E1. (c) OASPL vs. yaw angle for the elliptical cylinder E2. (d) Bandwidth vs. yaw angle for the elliptical cylinder E2.

using the correction factors proposed by the independence principle or those proposed in the present study is given.

Table 1 lists the normal Strouhal number $S t_{n}$ averaged over both flow speed and yaw angle for each of the cylinder cross-sections. The standard deviation $\sigma$ shows the dispersion in the results. For consistency across cylinder types, the results for the circular cylinder at a yaw angle of $75^{\circ}$ are not included.

In the previous sections it was shown how the Strouhal number varies with the yaw angle for circular, square, rectangular and elliptical cylinders. The results have been compared with the factor $\cos (\beta)$ and good agreement was 
Table 1: Average $S t_{n}$ obtained for each of the cylinder cross-sections. The standard deviation $\sigma$ shows the dispersion of the results.

\begin{tabular}{|c|c|c|c|c|c|c|}
\hline & \multicolumn{6}{|c|}{ Cylinder identifier } \\
\hline & $\mathrm{C}$ & $\mathrm{S}$ & $\mathrm{R} 1$ & $\mathrm{R} 3$ & $\mathrm{E} 1$ & $\mathrm{E} 2$ \\
\hline average $S t_{n}$ & 0.170 & 0.123 & 0.107 & 0.123 & 0.185 & 0.209 \\
\hline$\sigma$ & 0.016 & 0.005 & 0.004 & 0.008 & 0.007 & 0.018 \\
\hline
\end{tabular}

found in most cases. Figure 15(a) shows the absolute error obtained applying the factor $\cos (\beta)$ with respect to the values of average Strouhal number (for five flow speeds) measured for the different cylinder cross-sections and yaw angles. The error obtained is less than $12.5 \%$ except for the yaw angle of $75^{\circ}$. In this case the Strouhal number rises significantly, as discussed earlier in section 3.1, so the Strouhal number is not well predicted by the factor $\cos (\beta)$. It is not clear if this disagreement corresponds to a limitation on the maximum angle for which the independence principle can be used or whether the measured results are affected by the experimental arrangement.
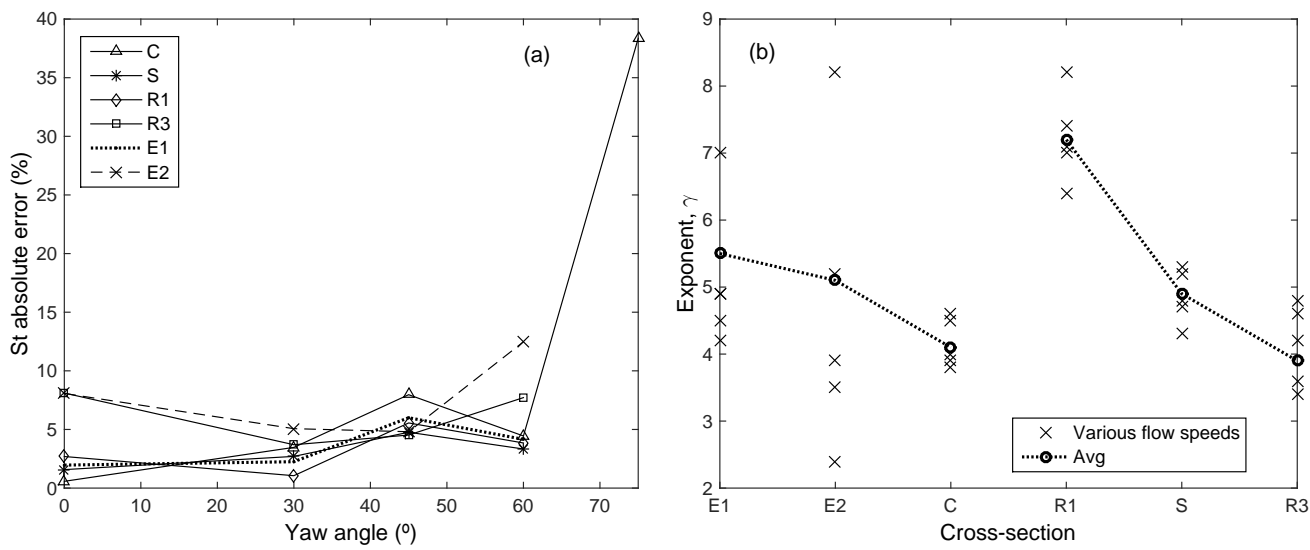

Figure 15: (a) Absolute error (\%) obtained from the comparison of the theoretical factor $(\cos \beta)$ and the results measured for the variation of the average St (for five flow speeds) with the yaw angle, for each of the cylinder cross-sections. (b) Exponent $\gamma$ that provides the smallest error between the measurements of OASPL and the calculations using the factor $10 \log _{10}\left(\cos ^{\gamma}(\beta)\right)$. The results are shown for each of the yaw angles and cylinder cross-sections.

The variation of the SPL with the yaw angle can be roughly predicted by using a factor of $10 \log _{10}\left(\cos ^{\gamma}(\beta)\right)$, according to the independence principle. For each of the cylinder cross-sections and flow speeds the exponent $\gamma$ that provides the lowest absolute error compared with the measurements was assessed for the different yaw angles. The results are shown in Figure 15(b) for each of the flow speeds. The average value of $\gamma$, also shown in Figure 15(b), was obtained by using least squares to the results of the measurements averaged for the five flow speeds.

The results in Figure 15(b) show that a unique value of the exponent $\gamma$ cannot be applied and a different exponent $\gamma$ should be considered for each of the cylinder cross-sections. Moreover, a large variability of the value of the exponent $\gamma$ was found for each of the cylinder cross-sections and different flow speeds.

\section{Speed exponent}

Figure 16(a) shows the variation of the OASPL with speed, in the form of the Reynolds number, for cylinders with different cross-sections at a yaw angle of $0^{\circ}$. The cylinder R3 is not shown as it did not generate vortex shedding at zero yaw angle. The speed exponent $\alpha$, shown in Figure 16(a) for each of the cylinder cross-sections, was obtained from the slope of the straight line given by a linear curve fit to the results. There are significant variations in the values of $\alpha$ for the different cylinder cross-sections ranging from 5.1 to 6.5 .

It should be noted that the characteristic dimension $D$ of each of the cylinder cross-sections is different. Although they are plotted against Reynolds number, the SPL has not been corrected for this dimension. The purpose of Figure 
16(a) is to compare the dependence on flow speed of cylinders with different cross-sections, thus the OASPL for different cylinder cross-sections should not be compared directly in this figure.

To allow that comparison, Figure 16(b) shows the OASPL measured for each of the cylinder cross-sections for different flow speeds. In these results, the measured OASPL has been corrected to account for the effect of the differences in the value $D$ for the different cylinder cross-sections using Eq. (4). Despite the differences in Reynolds number range covered for the different cylinder cross-sections, due to the different values of $D$ (see Table A.1), it is assumed that no significant changes in the flow behaviour are experienced. The quietest cylinders are the elliptical ones with OASPL between 12 and $15 \mathrm{~dB}$ lower than that from the square cylinder (the noisiest). The rectangular cylinder R4 is between 5 and $7 \mathrm{~dB}$ quieter than the square cylinder (although note that its aspect ratio is small). The rectangular cylinders R1 and R2 are only slightly quieter than the square cylinder. The circular cylinder is significantly quieter than the square and rectangular cylinders for low flow speeds. However, due to a higher speed dependence, for flow speeds of 40 and $50 \mathrm{~m} / \mathrm{s}$ it is within $2 \mathrm{~dB}$ of the square cylinder.
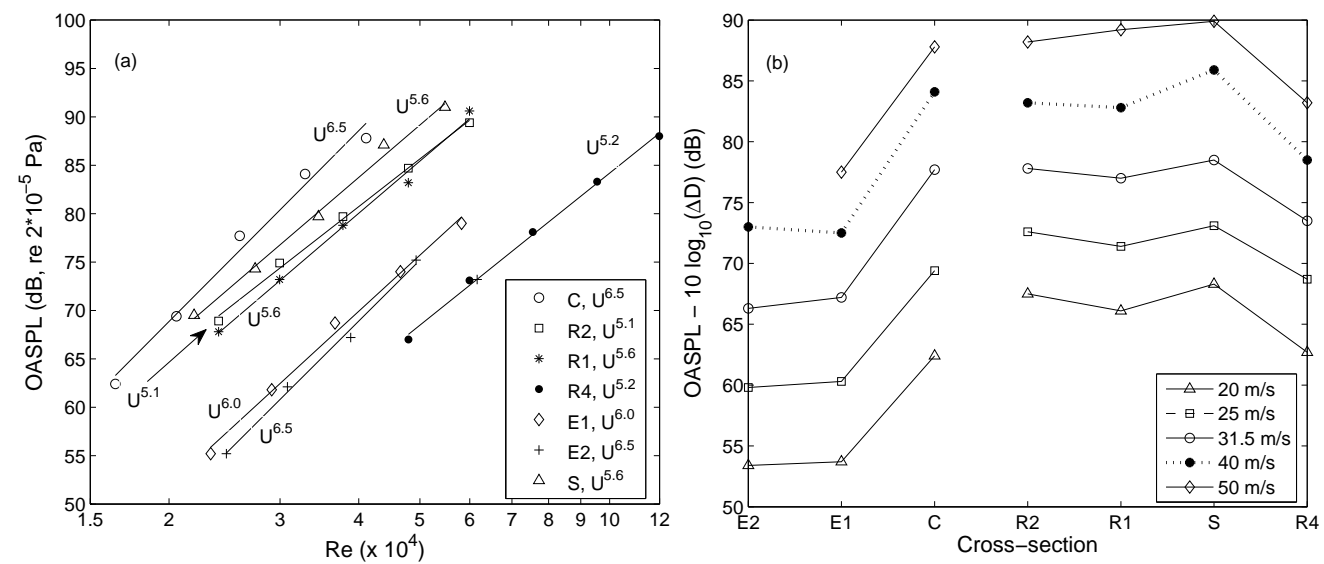

Figure 16: Speed dependence of the noise level radiated by cylinders with different cross-sections. (a) Variation of the OASPL with the Reynolds number (for increasing flow speeds). The speed exponents obtained for each of the cylinders are indicated. (b) OASPL obtained for the different cylinder cross-section shapes for different flow speeds. The correction given in Eq. (4) was applied to account for the effect of different characteristic dimension $D$ for the different cylinder cross-sections.

\section{Dependence on the angle of attack for square cylinders}

The influence of the angle of attack has been studied for the square cylinder. Figure 17 shows the narrow-band noise spectra measured for the square cylinder at different angles of attack. The results show a small reduction of the peak amplitude of the noise spectrum at the vortex shedding frequency between $0^{\circ}$ and $10^{\circ}$. The decrease of the SPL is significant when the angle of attack is increased to $15^{\circ}$ showing that coherent vortex shedding is no longer triggered above a certain angle of attack between $10^{\circ}$ and $15^{\circ}$, and for higher angles the noise spectrum is broadband.

These results are in agreement with other experimental noise measurements found in the literature for similar Reynolds numbers (see Table A.7). Yamada et al. [19] measured the noise radiated by a square cylinder for different angles of attack between $-45^{\circ}$ and $45^{\circ}$. They found a steep decrease of the SPL (of around $19 \mathrm{~dB}$ ) and an increase of the Strouhal number (up to 0.148 ) for angles of attack between $10^{\circ}$ and $15^{\circ}$, indicating changes in the flow behaviour. Similar results were found by Tamura and Miyagi [34] who found that for yaw angles between $10^{\circ}$ and $15^{\circ}$ the measured rms fluctuating lift coefficient decreased significantly and the Strouhal number increased up to 0.152 .

Other experimental results are available in the literature in terms of fluctuating lift coefficient. The effect on noise can be estimated by assuming that the corresponding decrease of the SPL can be calculated from Eq. (2) using a factor $20 \log _{10}\left(\Delta C_{L r m s}\right)$, where $\Delta C_{L r m s}$ is the ratio between the rms fluctuating lift coefficient for a certain angle of attack $\vartheta$ and for $\vartheta=0^{\circ}$. A decrease of around $15 \mathrm{~dB}$ is obtained from the results in terms of $C_{\text {Lrms }}$ obtained by Tamura and Miyagi [34]. A similar decrease of the rms fluctuating lift coefficient was obtained by Knisely [10] for angles of 


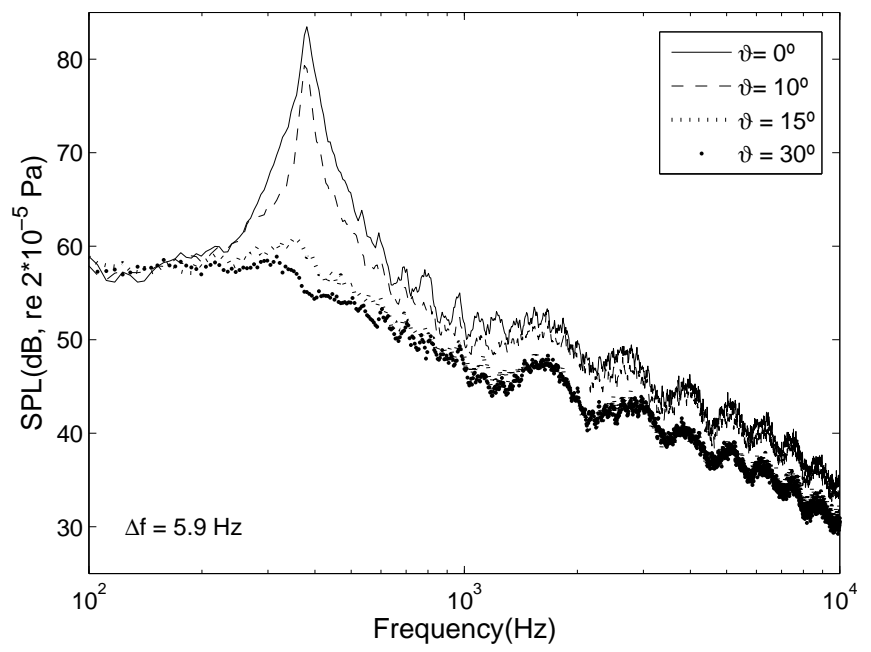

Figure 17: Noise spectra radiated by the square cylinder at different angles of attack $\vartheta$ for a Reynolds number of $5.48 \times 10^{4}($ flow speed of $50 \mathrm{~m} / \mathrm{s})$.

attack between $10^{\circ}$ and $15^{\circ}$. If the same factor is used an equivalent noise reduction of $17 \mathrm{~dB}$ is obtained. Knisely also found an increase of the Strouhal number up to 0.171 was measured for angles of attack above $15^{\circ}$. These results are in good agreement with those obtained in the present experiments and those from noise measurements found in the literature.

However, the results found in the literature show an increase of the SPL and variations of Strouhal number for angles higher than $15^{\circ}$, indicating that a vortex shedding peak was still detected, while in the present experiments the vortex shedding peak was not triggered for angles higher than $15^{\circ}$.

\section{Dependence on the cross-sectional shape}

In this section the effects of cross-sectional shape are summarised for elliptical and rectangular cylinders by comparing the Strouhal number and OASPL with the corresponding results for a circular or square cylinder respectively.

\subsection{Eccentricity of elliptical cylinders}

The elliptical cylinders are defined in terms of their eccentricity $e=\sqrt{\left(1-(D / B)^{2}\right)}$ (see Figure 2) where $e=0$ for a circular cylinder. Figure 18(a) shows the Strouhal number obtained for elliptical cylinders with various values of the eccentricity from the current work and from the literature ([12], [35] and [14]). The Strouhal numbers from the current tests are the average over the five flow speeds. The corresponding aspect ratios and Reynolds numbers from the present tests and the experimental works found in the literature are listed in Tables A.1, A.2 and A.7. The variation of the Strouhal number with the flow speed in this Reynolds number range was found to be small, as shown in Figure 13. All the results are shown for an angle of attack of $0^{\circ}$.

The results show relatively constant Strouhal numbers for eccentricities between 0 and 0.8 (values of $S t$ between 0.17 and 0.21 ). For eccentricities above 0.8 the Strouhal number increases up to a value of 0.480 for an eccentricity of 0.97 [35]. The results from the present experiments are quite consistent, for the Reynolds number range considered, with those from Modi and Wiland [12], Fujita [35] and King and Pfizenmaier [14]. However, for higher Reynolds numbers $\left(1.3 \times 10^{5}\right)$ King and Pfizenmaier found a significant increase of the Strouhal number measured for the elliptical cylinder with eccentricity of 0.86 , with values of 0.374 for an aspect ratio of 16 and 0.407 for an aspect ratio of 25 [14].

Figure 18(b) shows the difference between the noise level radiated by an elliptical cylinder and by a circular cylinder for different eccentricities. The results have been corrected to account for the different values of $D$ for the different cylinder cross-sections by means of Eq. (4). The present results are again compared with results from the 

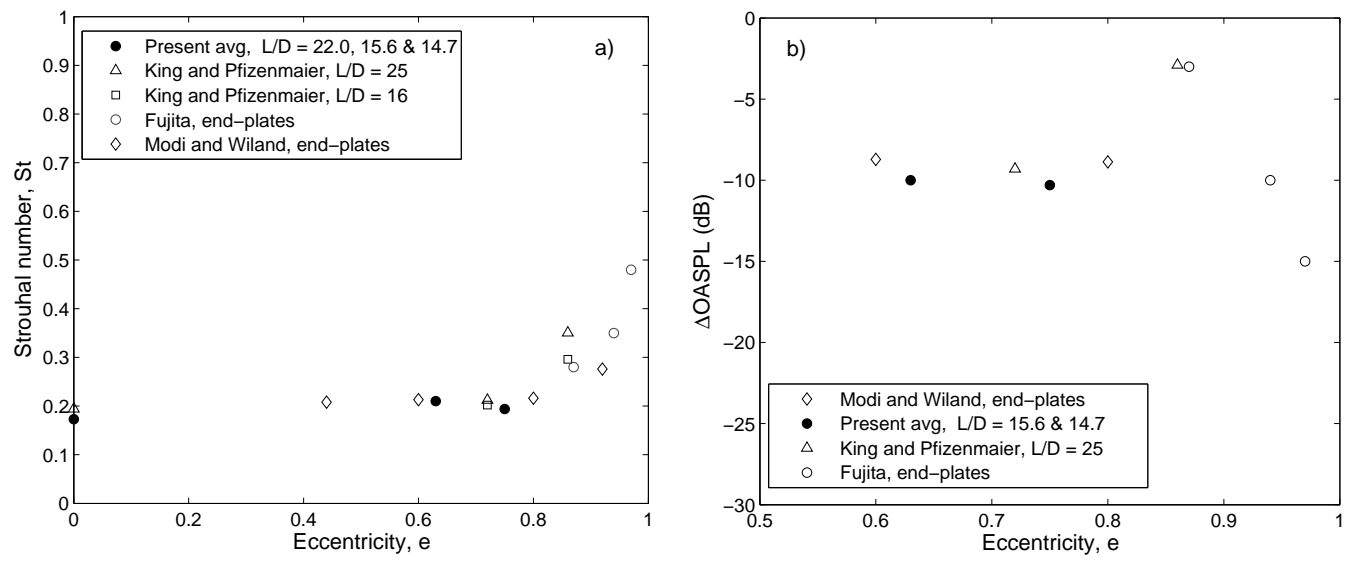

Figure 18: Influence of the eccentricity of elliptical cylinders on St and SPL. (a) Variation of the Strouhal number St with the cylinder eccentricity. (b) Variation of the difference between the noise radiated by an elliptical cylinder and by a circular cylinder for different eccentricities of the elliptical cylinder. The results have been corrected to account for the different values of $D$ by means of Eq. (4). Results include experiments carried out during the present work, results from Modi and Wiland [12], Fujita [35] and King and Pfizenmaier [14]. Reynolds numbers are given in Table A. 1 and Table A.7.

literature. The data obtained by King and Pfizenmaier are only shown for an aspect ratio of 25. For an aspect ratio of 16 they found significant three dimensional effects leading to smaller differences between the SPL from the elliptical and circular cylinders [14]. They also found a steep increase of the SPL for the cylinder with eccentricity of 0.86 and a Reynolds number $1.3 \times 10^{5}$, the noise from the elliptical cylinder being $3 \mathrm{~dB}$ lower than that from the circular cylinder. The noise reduction obtained from the data measured by Modi and Wiland [12] was estimated using a factor $20 \log _{10}\left(\Delta C_{\text {Lrms }}\right)$ where $\Delta C_{\text {Lrms }}$ is the ratio between the rms fluctuating lift coefficient measured for an elliptical cylinder and for a circular cylinder.

Reductions of around $10 \mathrm{~dB}$ with respect to the noise radiated by a circular cylinder are found for most values of eccentricity. For eccentricities of around 0.86 both Fujita [35] and King and Pfizenmaier [14] found similar results with reductions of around $3 \mathrm{~dB}$.

\subsection{Slenderness ratio of rectangular cylinders}

For rectangular cylinders, the Strouhal number is plotted against the slenderness ratio $(B / D)$ in Figure 19(a). Results obtained by King and Pfizenmaier [14] and Knisely [10] are also shown for comparison. The latter results were obtained from the fluctuating velocity in the cylinder wake. The Reynolds number range covered, listed in Table A.1 and Table A.7, was slightly different in the different experiments but it can be assumed that the flow behaviour remained unchanged. For all the cases the results were nearly constant with the flow speed so averaged results are shown.

The results in Figure 19(a) show a decrease of the Strouhal number with cylinder slenderness ratio. The results included from the different investigations show a good agreement except for $B / D=0.5$, for which the value obtained in the present work $(S t=0.167)$ is higher than that obtained by Knisely $(S t=0.140)$. The dotted line, obtained by fitting a straight line against $\log (B / D)$, shows the variation of $S t$ with the slenderness ratio for a range $0.5<B / D<2.5$.

Figure 19(b) shows the OASPL radiated by rectangular cylinders with different slenderness ratios normalised by the result for a square cylinder, the results also being corrected for differences in $D$ by means of Eq. (4). The results obtained in the present study are also compared with those obtained by King and Pfizenmaier [14]. The results show slight variations of the OASPL (less than $+/-2 \mathrm{~dB}$ ) for all the slenderness ratios considered except for $B / D=0.5$, where the difference is $-6.7 \mathrm{~dB}$. Knisely found a decrease of the rms fluctuating lift coefficient from 1 for a square cylinder to 0.6 for a rectangular cylinder with $B / D=0.5$. According to Eq. (2) this leads to a reduction of the noise of $-4.4 \mathrm{~dB}$, showing good agreement with the results obtained in the present studies. 

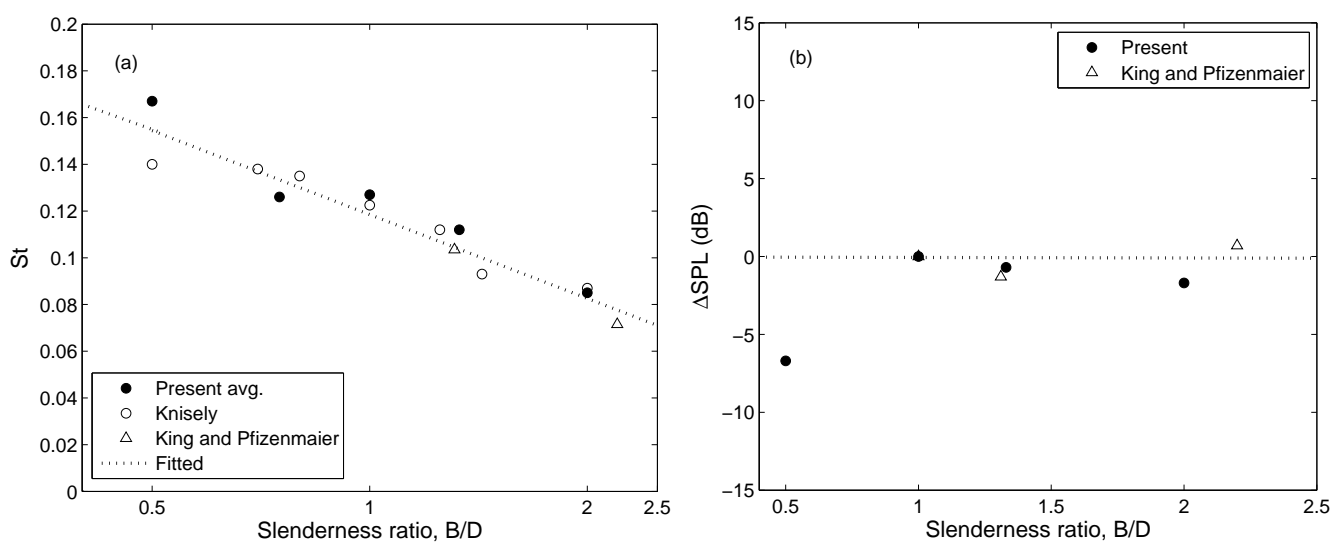

Figure 19: Effect of the slenderness ratio of a rectangular cylinder on the vortex shedding noise. Results include present investigations and those from King and Pfizenmaier [14] and Knisely [10]. Reynolds numbers are given in Table A.1 and Table A.7. (a) Variation of the Strouhal number $S t$ with the slenderness ratio $B / D$. (b) SPL radiated by rectangular cylinders with different slenderness ratios $B / D$ normalised to the result for a square cylinder $(B / D=1)$. The results have been corrected to account for the different values of $D$ by means of Eq. (4).

From the results shown in Figure 16(a), the speed exponent $\alpha$ of rectangular cylinders with different slenderness ratio was obtained. This is shown in Figure 20. The maximum speed exponent $(\alpha=5.6)$ is obtained for the square cylinder; the speed exponent decreases slightly for rectangular cylinders. The effect of the different aspect ratios $L / D$ for the different rectangular cylinders has not been accounted for.

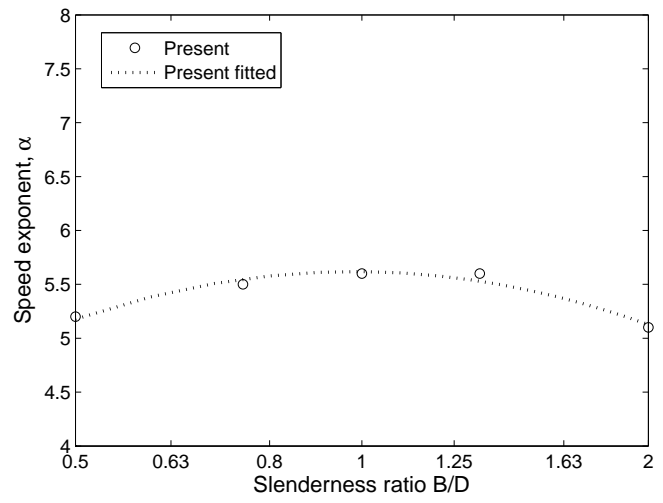

Figure 20: Variation of the speed exponent $\alpha$ with the slenderness ratio of rectangular cylinders obtained during the present work.

\section{Directivity}

The directivity was measured using the microphone grid shown in Figure 3. For simplicity in the presentation of the results no correction was applied to the radiation angle to account for the effect of sound convection and shear layer refraction. The maximum angle correction (for a flow speed of $50 \mathrm{~m} / \mathrm{s}$ ) obtained by using Amiet's method [31] was not significant (of around $3^{\circ}$ ). However, the amplitude of the measured noise was corrected for convective amplification as explained in section 2.2.8. The results are presented for circular and square cylinders. Similar results were found for other geometries [23].

Figure 21(a) shows the directivity measured for the circular cylinder in terms of OASPL. The directivity for all the flow speeds and the average directivity are represented with markers. These results are compared with the directivity 
for a theoretical dipole source. Good agreement is found, with maximum differences of $2 \mathrm{~dB}$ between the measured and theoretical values. These results agree with those obtained by Porteous et al. [16] and Hutcheson and Brooks [17]. For some of the radiation angles the dispersion in the results is large, around $+/-3 \mathrm{~dB}$. A good agreement is found if the results from the two different microphone arrays are compared (for $45^{\circ}$ and $60^{\circ}$ ) confirming the symmetry in the noise radiation between the longitudinal and transverse planes with respect to the flow direction. Although the pressure from a theoretical dipole source tends to zero at $90^{\circ}$, the measured results are limited to around 15-20 dB below the level for the normal direction due to contributions from the broadband component and the background noise.
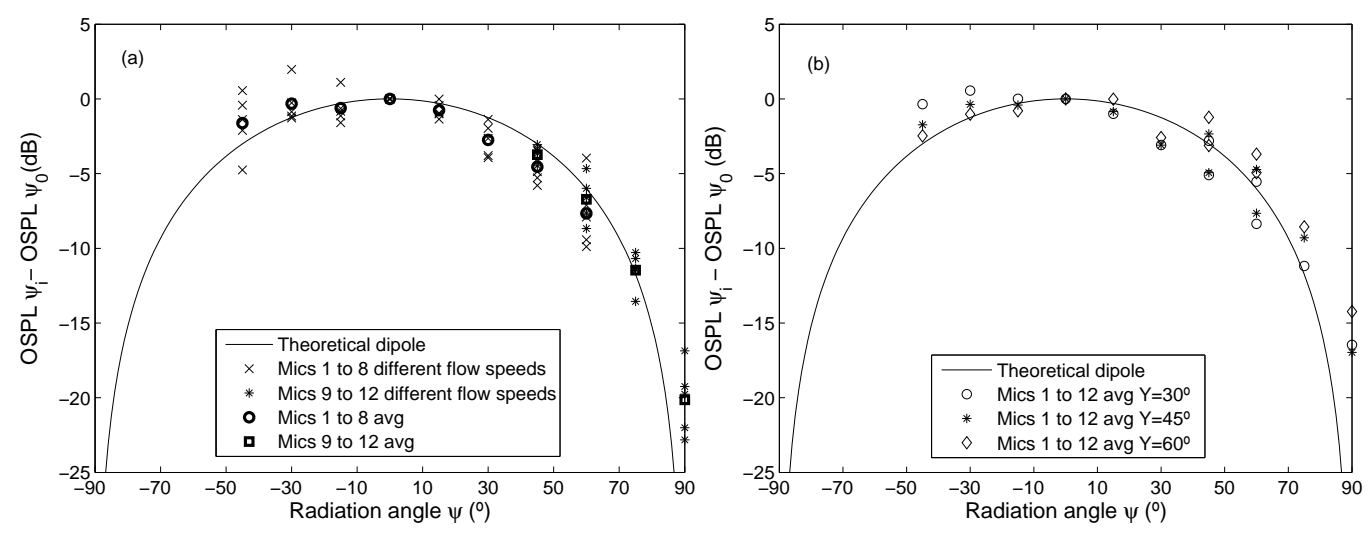

Figure 21: Directivity of the circular cylinder. The results are compared with the directivity of a theoretical dipole source. (a) Directivity for the circular cylinder normal to the flow. Averaged results are shown together with the results for different five flow speeds. (b) Directivity for the circular cylinder yawed by different angles. Results were averaged for different flow speeds.

Figure 21(b) shows the directivity for the circular cylinder yawed by different angles. These results are averaged over speed. From this it seems that the directivity of the vortex shedding noise is independent of the yaw angle. The measurements made using the transverse array, mainly for the higher yaw angles, are slightly higher than the results measured by the longitudinal array, the latter following the directivity of the theoretical dipole source more closely. The results for $75^{\circ}$ are not included in the figure as the cylinder does not satisfy the compact source conditions in this case.

The directivity for the square cylinder normal to the flow is shown in Figure 22(a) for different flow speeds as well as the average value. The trend followed is again close to the directivity for a theoretical dipole, with a small dispersion in the results for the angles close to the central positions and an increasing dispersion for further angles. Figure 22(b) shows the directivity of the square cylinder for different yaw angles. This is also in good agreement with that for a theoretical dipole. In this case the results of the transverse array are around $5 \mathrm{~dB}$ higher than expected.

\section{Conclusions}

The results obtained from anechoic wind tunnel tests using cylinders with different cross-sections (circular, square, rectangular and elliptical) are presented. These are used to assess the dependence of the vortex shedding noise on the yaw angle, flow speed, cross-sectional shape (eccentricity and slenderness ratio), angle of attack (for a square cylinder) and directivity for Reynolds numbers in the range from $1.6 \times 10^{4}$ to $1.2 \times 10^{5}$.

The amplitude and frequency of the vortex shedding noise was found to decrease with increasing yaw angle. Good agreement was found with the independence principle for the variation of the Strouhal number for all the cylinder cross-sections, except for the circular cylinder yawed by an angle of $75^{\circ}$. In terms of the variation of the Overall Sound Pressure Level (OASPL) with the yaw angle the results do not follow the independence principle. Alternative correction factors are proposed for each of the cylinder cases, with exponents both greater and less than 6 . The relative bandwidth of the vortex shedding peak was found to increase with the yaw angle for all the cylinder cross-sections except for the elliptical cylinders. 

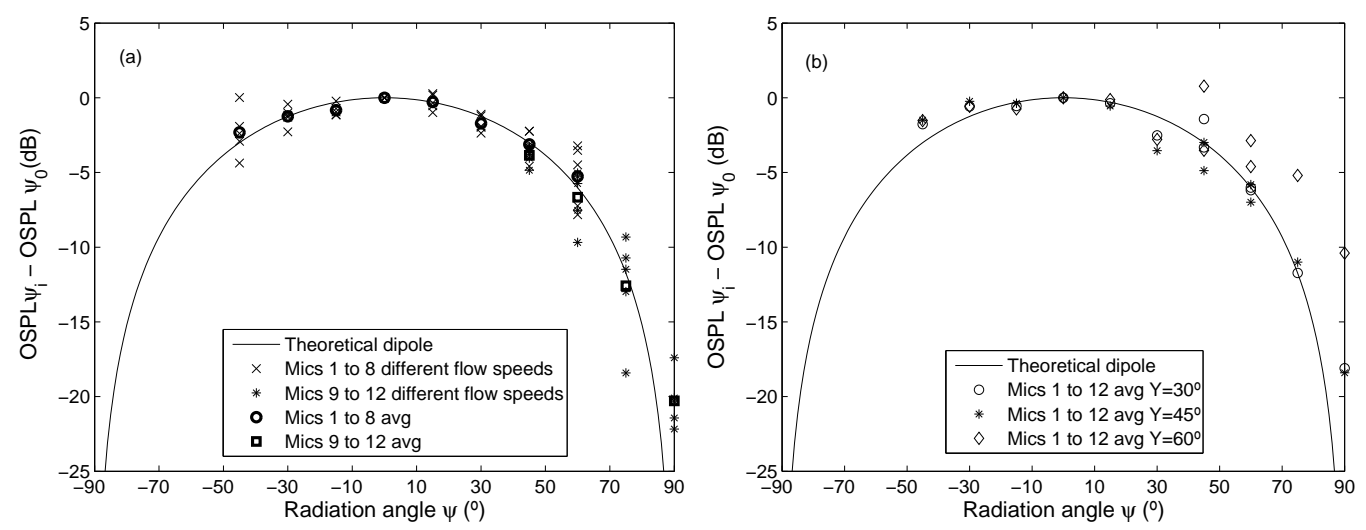

Figure 22: Directivity of the square cylinder. The results are compared with the directivity of a theoretical dipole source. (a) Directivity for the square cylinder normal to the flow. Averaged results are shown together with the results for different five flow speeds. (b) Directivity for the square cylinder yawed by different angles. Results were averaged for different flow speeds.

The cross-sectional shape of the cylinder was found to affect the Strouhal number, amplitude and speed dependence of the vortex shedding noise. The square cylinder was found to be the noisiest and the elliptical cylinders the quietest, while the highest speed exponent $\alpha$ was found for the circular cylinder and the lowest for the rectangular cylinder with slenderness ratio 2 , all of them being in the range $5.1 \leq \alpha \leq 6.5$. The Strouhal number and noise level (relative to the circular cylinder) was nearly constant for the elliptical cylinders with eccentricities 0.63 and 0.75 . The Strouhal number decreased with increasing slenderness ratio of the assessed rectangular cylinders and the noise level was found to be similar to that from the square cylinder, except for $B / D=0.5$ which gave a level $6.7 \mathrm{~dB}$ lower.

For the square cylinder the vortex shedding was no longer triggered for angles of attack of $15^{\circ}$ and above. This result is in good agreement with those found in the literature.

The directivity of the vortex shedding noise radiated by circular and square cylinders was found to be close to that expected for a theoretical dipole source, with differences less than $+/-3 \mathrm{~dB}$. The cylinder directivity was found to be nearly independent of the yaw angle; symmetry was found in the results between the longitudinal and transverse planes.

\section{Appendix A. Additional data of the experimental set-up and Reynolds number range covered}

Table A.1 shows the Reynolds number range covered during the experiments for the different cylinder crosssections. The characteristic dimension $(D)$ used for each of the cylinders is also listed. Although the characteristic dimension of all the cylinders was kept constant during the tests, the effective length of the cylinder has increased with the increase of the yaw angle.

Table A.2 shows the aspect ratio $(L / D)$ and length $(L)$ for each of the yaw angles and for each of the cylinders used. The effective length was determined from the total length of the cylinder in the air stream, excluding the part within the shear layer.

Table A. 3 shows the maximum and minimum vortex shedding frequency $\left(f_{0}\right)$ and wavelength $(\lambda)$ obtained for each of the cylinders. The maximum vortex shedding frequency is obtained for the maximum flow speed $(50 \mathrm{~m} / \mathrm{s})$ and the minimum yaw angle $\left(0^{\circ}\right)$ while the minimum vortex shedding frequency is obtained for the minimum flow speed $(20 \mathrm{~m} / \mathrm{s})$ and the maximum yaw angle $\left(60^{\circ}, 75^{\circ}\right.$ in the case of the circular cylinder).

Table A.4 shows the effective length of the cylinders for the different yaw angles and the correction applied to the SPL radiated by the cylinders to account for the increase of the effective length with the yaw angle (Eq. (3)).

The convective amplification was corrected using the theoretical factor $(1-M \cos (\phi))^{4}$, as shown in Eq. (2). Table A. 5 shows the corrections applied for each of the microphones of the longitudinal array (microphones 1 to 8 ). No correction was applied for the microphones of the transverse array. 
Table A.1: Reynolds number based on $U_{\infty}$ and $D$ for the different cylinder cross-sections and flow speeds used. The characteristic dimension $D$ is expressed in $\mathrm{mm}$.

\begin{tabular}{|c|c|c|c|c|c|c|c|}
\hline \multicolumn{9}{|c|}{ Reynolds number } \\
\hline \multicolumn{3}{|c|}{ Cylinder } & \multicolumn{5}{c|}{ Flow speeds $(\mathrm{m} / \mathrm{s})$} \\
\hline Cross-section & Identifier & $\mathrm{D}(\mathrm{mm})$ & 20 & 25 & 31.5 & 40 & 50 \\
\hline Circular & $\mathrm{C}$ & 12.0 & $1.64 \times 10^{4}$ & $2.05 \times 10^{4}$ & $2.59 \times 10^{4}$ & $3.29 \times 10^{4}$ & $4.11 \times 10^{4}$ \\
\hline Square & $\mathrm{S}$ & 16.0 & $2.19 \times 10^{4}$ & $2.74 \times 10^{4}$ & $3.45 \times 10^{4}$ & $4.38 \times 10^{4}$ & $5.48 \times 10^{4}$ \\
\hline Rectangular & R1 \& R2 & 17.5 & $2.40 \times 10^{4}$ & $3.00 \times 10^{4}$ & $3.78 \times 10^{4}$ & $4.79 \times 10^{4}$ & $5.99 \times 10^{4}$ \\
\hline Rectangular & R3 & 23.3 & $3.19 \times 10^{4}$ & $3.99 \times 10^{4}$ & $5.03 \times 10^{4}$ & $6.37 \times 10^{4}$ & $7.97 \times 10^{4}$ \\
\hline Rectangular & R4 & 35.0 & $4.80 \times 10^{4}$ & $6.00 \times 10^{4}$ & $7.56 \times 10^{4}$ & $9.58 \times 10^{4}$ & $1.20 \times 10^{5}$ \\
\hline Elliptical & E1 & 17.0 & $2.33 \times 10^{4}$ & $2.91 \times 10^{4}$ & $3.67 \times 10^{4}$ & $4.66 \times 10^{4}$ & $5.82 \times 10^{4}$ \\
\hline Elliptical & E2 & 18.0 & $2.47 \times 10^{4}$ & $3.08 \times 10^{4}$ & $3.88 \times 10^{4}$ & $4.93 \times 10^{4}$ & $6.16 \times 10^{4}$ \\
\hline
\end{tabular}

Table A.2: Aspect ratio $(L / D)$ and effective length $(L)$ for each of the cylinder cross-sections and yaw angles used during the experiments. The thickness of the shear layer has been subtracted at both ends (assuming same thickness) to give the length of the cylinder in the clean air flow.

\begin{tabular}{|c|c|c|c|c|c|c|c|}
\hline \multicolumn{3}{|c|}{ Aspect ratio $(L / D)$} & \multicolumn{5}{|c|}{ Yaw angle $(\beta)$} \\
\hline Cross-section & Identifier & $\mathrm{D}(\mathrm{mm})$ & 0 & 30 & 45 & 60 & 75 \\
\hline Circular & $\mathrm{C}$ & 12.0 & 22.0 & 26.5 & 34.1 & 51.2 & 105.5 \\
\hline Square & $\mathrm{S}$ & 16.0 & 16.5 & 19.9 & 25.6 & 38.4 & - \\
\hline Rectangular & R1 \& R2 & 17.5 & 15.1 & 18.2 & 23.4 & 35.1 & - \\
\hline Rectangular & $\overline{\mathrm{R} 3}$ & 23.3 & 11.3 & 13.6 & 17.5 & 26.3 & - \\
\hline Rectangular & $\mathrm{R} 4$ & 35.0 & 7.5 & 9.1 & 11.7 & 17.5 & - \\
\hline Elliptical & E1 & 17.0 & 15.6 & 18.7 & 24.1 & 36.1 & - \\
\hline Elliptical & E2 & 18.0 & 14.7 & 17.7 & 22.7 & 34.1 & - \\
\hline \multicolumn{3}{|c|}{ Effective length $L(\mathrm{~cm})$} & 26.4 & 31.8 & 40.9 & 61.4 & 126.6 \\
\hline
\end{tabular}

Table A.3: Maximum and minimum vortex shedding frequency $\left(f_{0}\right)$ and corresponding wavelength $(\lambda)$ measured for the different cylinders. The percentage of cases that satisfy the far-field and compact source conditions are given for each of the cylinder cross-sections.

\begin{tabular}{|c|c|c|c|c|c|c|c|}
\hline & Max. $f_{0}(\mathrm{~Hz})$ & Min. $f_{0}(\mathrm{~Hz})$ & Min. $\lambda(\mathrm{m})$ & Max. $\lambda(\mathrm{m})$ & $R>2 L$ & $R>\lambda$ & $L<\lambda$ \\
\hline C & 755 & 105 & 0.45 & 3.24 & $80 \%$ & $68 \%$ & $80 \%$ \\
\hline S & 386 & 76 & 0.88 & 4.47 & $100 \%$ & $30 \%$ & $100 \%$ \\
\hline E1 & 539 & 111 & 0.63 & 3.05 & $100 \%$ & $67 \%$ & $100 \%$ \\
\hline E2 & 978 & 111 & 0.35 & 3.00 & $100 \%$ & $56 \%$ & $100 \%$ \\
\hline R1 & 310 & 82 & 1.09 & 4.15 & $100 \%$ & $15 \%$ & $100 \%$ \\
\hline R3 & 216 & 58 & 1.57 & 5.80 & $100 \%$ & $0 \%$ & $100 \%$ \\
\hline
\end{tabular}

Table A.4: SPL correction (expressed in $\mathrm{dB}$ ) to account for the increase of the effective length of the cylinder $L$ with the increase of the yaw angle.

\begin{tabular}{|c|c|c|c|c|c|}
\hline & \multicolumn{5}{|c|}{ Yaw angle $(\beta)$} \\
\hline & $0^{\circ}$ & $30^{\circ}$ & $45^{\circ}$ & $60^{\circ}$ & $75^{\circ}$ \\
\hline Length $L(\mathrm{~cm})$ & 26.4 & 31.8 & 40.9 & 61.4 & 126.6 \\
\hline Correction $(\mathrm{dB})$ & 0.0 & -0.6 & -1.5 & -3.0 & -5.9 \\
\hline
\end{tabular}


Table A.5: Convective amplification correction (in $\mathrm{dB}$ ) for each of the microphones of the longitudinal array.

\begin{tabular}{|c|c|c|c|c|c|c|}
\hline \multicolumn{2}{|c|}{} & \multicolumn{5}{|c|}{ Flow speed (m/s) } \\
\hline Mic. number & $\phi$ & 20 & 25 & 31.5 & 40 & 50 \\
\hline 1 & $135^{\circ}$ & -0.7 & -0.8 & -1.1 & -1.4 & -1.8 \\
\hline 2 & $120^{\circ}$ & -0.5 & -0.6 & -0.8 & -1.0 & -1.3 \\
\hline 3 & $105^{\circ}$ & -0.3 & -0.3 & -0.4 & -0.5 & -0.7 \\
\hline 4 & $90^{\circ}$ & 0.0 & 0.0 & 0.0 & 0.0 & 0.0 \\
\hline 5 & $75^{\circ}$ & 0.3 & 0.3 & 0.4 & 0.5 & 0.7 \\
\hline 6 & $60^{\circ}$ & 0.5 & 0.6 & 0.8 & 1.1 & 1.4 \\
\hline 7 & $45^{\circ}$ & 0.8 & 0.9 & 1.1 & 1.5 & 1.9 \\
\hline 8 & $30^{\circ}$ & 0.9 & 1.1 & 1.4 & 1.9 & 2.4 \\
\hline
\end{tabular}

Table A.6 show the corrections applied to each of the microphones of the longitudinal array (microphones 1 to 8) to account for the effect of the shear layer refraction, according to the method proposed by Amiet [31]. For the transverse array a negligible influence of the shear layer was assumed.

Table A.6: Shear layer refraction correction (in $\mathrm{dB}$ ) for each of the microphones of the longitudinal array.

\begin{tabular}{|c|c|c|c|c|c|c|}
\hline \multicolumn{2}{|c|}{} & \multicolumn{5}{|c|}{ Flow speed (m/s) } \\
\hline Mic. number & $\phi$ & 20 & 25 & 31.5 & 40 & 50 \\
\hline 1 & $135^{\circ}$ & 1.1 & 1.3 & 1.7 & 2.1 & 2.6 \\
\hline 2 & $120^{\circ}$ & 0.7 & 0.9 & 1.1 & 1.4 & 1.7 \\
\hline 3 & $105^{\circ}$ & 0.4 & 0.4 & 0.5 & 0.7 & 0.9 \\
\hline 4 & $90^{\circ}$ & 0.0 & 0.0 & 0.0 & 0.0 & 0.0 \\
\hline 5 & $75^{\circ}$ & -0.3 & -0.4 & -0.5 & -0.6 & -0.8 \\
\hline 6 & $60^{\circ}$ & -0.7 & -0.8 & -1.1 & -1.4 & -1.7 \\
\hline 7 & $45^{\circ}$ & -1.1 & -1.4 & -1.7 & -2.2 & -2.8 \\
\hline 8 & $30^{\circ}$ & -1.8 & -2.3 & -2.7 & -3.1 & -3.5 \\
\hline
\end{tabular}

Table A.7 shows the Reynolds number and aspect ratio range covered during the experiments found in the literature. 
Table A.7: Reynolds number $R e$ and aspect ratio $(L / D)$ range covered during the experiments found in the literature.

\begin{tabular}{|c|c|c|}
\hline Authors & Reynolds number $R e$ & Aspect ratio $(L / D)$ \\
\hline \multicolumn{3}{|c|}{ Circular cylinder } \\
\hline Yamada et al. [19] & $1.4 \times 10^{4}-2.8 \times 10^{4}$ & end-plates \\
\hline Haramoto et al. [20] & $1.4 \times 10^{4}$ & end-plates \\
\hline King and Barsikow [21] & $6.7 \times 10^{4}$ & 25 (free-end) \\
\hline \multicolumn{3}{|c|}{ Square cylinder } \\
\hline Yamada et al. [19] & $1.4 \times 10^{4}-2.8 \times 10^{4}$ & end-plates \\
\hline King and Barsikow [21] & $1.0 \times 10^{5}$ & 25 (free-end) \\
\hline Tamura and Miyagi [34] & $3.0 \times 10^{4}$ & end-plates \\
\hline Knisely [10] & $2.2 \times 10^{4}-6.2 \times 10^{4}$ & end-plates \\
\hline \multicolumn{3}{|c|}{ Elliptical cylinder } \\
\hline Modi and Wiland [12] & $2.4 \times 10^{4}-1.0 \times 10^{5}$ & end-plates \\
\hline Modi et al. [13] & $3.0 \times 10^{4}-1.0 \times 10^{5}$ & end-plates \\
\hline Fujita [35] & $1.7 \times 10^{4}$ & end-plates \\
\hline King and Pfizenamier $e=0.72[14]$ & $9.5 \times 10^{4}$ & 16 and 25 (free-end) \\
\hline King and Pfizenamier $e=0.86[14]$ & $1.0 \times 10^{5}$ & 16 and 25 (free-end) \\
\hline \multicolumn{3}{|c|}{ Rectangular cylinder } \\
\hline Knisely [10] & $7.2 \times 10^{2}-8.1 \times 10^{4}$ & end-plates \\
\hline King and Pfizenamier [14] & $9.9 \times 10^{4}$ and $1.4 \times 10^{5}$ & 16.7 (free-end) \\
\hline
\end{tabular}

\section{References}

[1] O.M. Phillips, The intensity of Aeolian tones. Journal of Fluid Mechanics 1(6) (1956) 607-624. doi:10.1017/S0022112056000408

[2] B. Etkin, G.K. Korbacher, R.T. Keefe, Acoustic radiation from a stationary cylinder in a fluid stream (Aeolian tones). The Journal of the Acoustical Society of America 29(1) (1957) 3036. doi:10.1121/1.1908673

[3] R.T. Keefe, Investigation of the fluctuating forces acting on a stationary circular cylinder in a subsonic stream and of the associated sound field. The Journal of the Acoustical Society of America 34(11) (1962) 1711-1714. doi:10.1121/1.1909102

[4] H. Fujita, H. Furutani, H. Suzuki, Experimental investigations and prediction of aerodynamic sound generated from square cylinders, Proceedings of the Fourth AIAA/CEAS Aeroacoustics Conference, Tolousse, France, 1998, pp. 2369.

[5] N. Curle, The influence of solid boundaries upon aerodynamic sound. Proceedings of the Royal Society of London. Series A. Mathematical and Physical Sciences 231(1187) (1955) 505-514. doi:10.1098/rspa.1955.0191

[6] G. Schewe, On the force fluctuations acting on a circular cylinder in crossflow from subcritical up to transcritical Reynolds numbers. Journal of Fluid Mechanics 133(1) (1983) 265-285. doi:10.1017/S0022112083001913

[7] R.H. Schlinker, M.R.Fink, R.K.Amiet, Vortex noise from non-rotating cylinders and airfoils. Proceedings of the Forthteen Aerospace Science Meeting, Washington D.C., U.S.A., 1976, pp. 76-81.

[8] J.D. Hogan, J.W. Hall, Experimental study of pressure fluctuations from yawed circular cylinders. AIAA Journal 49(11) (2011) $2349-2356$. doi: $10.2514 / 1 . J 050302$

[9] B.J. Vickery, Fluctuating lift and drag on a long cylinder of square cross-section in a smooth and in a turbulent stream. Journal of Fluid Mechanics 25(3) (1966) 481-494. doi:10.1017/S002211206600020X

[10] C.W. Knisely, Strouhal numbers of rectangular cylinders at incidence: a review and new data. Journal of Fluids and Structures 4(4) (1990) 371-393. doi:10.1016/0889-9746(90)90137-T

[11] A. Okajima, Strouhal numbers of rectangular cylinders. Journal of Fluid Mechanics 123 (1982) 379-398. doi:10.1017/S0022112082003115

[12] V.J. Modi, E. Wiland, Unsteady aerodynamics of stationary elliptic cylinders in subcritical flow. AIAA Journal 10(8) (1969) 1814-1821. doi: $10.2514 / 3.5995$

[13] V.J. Modi, E. Wiland, A.K. Dikshitt, T. Yokomizo, On the fluid dynamics of elliptic cylinders. International Journal of Offshore and Polar Engineering 2(4) (1992) 267-280.

[14] W.F. King, E.Pfizenmaier, An experimental study of sound generated by flows around cylinders of different cross-section. Journal of Sound and Vibration 328(3) (2009) 318-337. doi:10.1016/j.jsv.2009.07.034

[15] D.J. Moreau, C.J. Doolan, Flow-induced sound of wall-mounted finite length cylinders. AIAA Journal 51(10) (2013) $2493-2502$. doi:10.2514/1.J052391

[16] R. Porteous, C.J. Doolan, D.J. Moreau, Directivity pattern of flow-induced noise from a wall-mounted, finite length circular cylinder, Proceedings of Acoustics, Victor Harbor, Australia, 2013.

[17] F. V. Hutcheson, T. F. Brooks, Noise radiation from single and multiple rod configurations. International Journal of Aeroacoustics 11(3) (2012) 291-334. doi:10.1260/1475-472X.11.3-4.291 
[18] M.M. Zdravkovich, Flow Around Circular Cylinders, vol. 1. Fundamentals (two volumes), Oxford University Press, Oxford, 1997.

[19] S. Yamada, H. Fujita, Y. Maruta, H. Maki, J. Shiraishi, Experimental Study on Aerodynamic Noise Generated from Two-Dimensional Models ( $2^{n} d$ Report, Effect of the angle of inclination of circular cylinders and the angle of attack of square cylinders to aerodynamic noise) (in Japanese). Trans. JSME 63(610) (1997) 1974-1979.

[20] Y. Haramoto, S. Yasuda, K. Matsuzaki, M. Munekata, Analysis of aerodynamic noise generated from inclined circular cylinder. Journal of Thermal Science 9(2) (2000) 122-128. doi:10.1007/s11630-000-0005-2

[21] W.F. King III, B. Barsikow, An experimental study of sound generated by flow interactions with cylinders, German-French cooperation project DEUFRAKO, Annex-K2, Final report, 1999.

[22] E. Latorre Iglesias, D.J. Thompson, M.G. Smith, Component-Based Model for Aerodynamic Noise of High-Speed Trains, Notes on Numerical Fluid Mechanics and Multidisciplinary Design 126 (2015) 481-488.

[23] E. Latorre Iglesias, D.J. Thompson, Wind tunnel tests on the noise radiated by cylinders with different cross-sections, Institute of Sound and Vibration Research Technical Memorandum 1002, 2014.

[24] T.P. Chong, P.F. Joseph, P. Davies, Design and performance of an open jet wind tunnel for aero-acoustic measurement. Applied Acoustics 70(4) (2009) 605-614. doi:10.1016/j.apacoust.2008.06.011

[25] R.D. Blevins, Flow-induced Vibration, Van Nostrand Reinhold Co., New York, 1977.

[26] T.J. Mueller, Aeroacoustic Measurements, Berlin, 2002.

[27] P.D. Welch, The use of fast Fourier transform for the estimation of power spectra: a method based on time averaging over short, modified periodograms. IEEE Transactions on audio and electroacoustics 15(2) (1967) 70-73. doi:10.1109/TAU.1967.1161901

[28] S.B. Pope, Turbulent Flows, Cambridge University Press, 2000.

[29] W. K. Blake, Mechanics of Flow-induced Sound and Vibration, vol. 1. General Concepts and Elementary Sources (two volumes), Academic Press, Orlando, Florida, 1986.

[30] P. Leehey, C.E. Hanson, Aeolian tones associated with resonant vibration. Journal of Sound and Vibration 13(4) (1970) 465-483. doi:10.1016/S0022-460X(70)80052-9

[31] R.K. Amiet, Refraction of sound by a shear layer. Journal of Sound and Vibration 58(4) (1978) 467-482. doi:10.1016/0022-460X(78)90353-X

[32] A.P. Dowling, J.E. Ffowcs Williams, Sound and Sources of Sound, Horwood, Chichester, 1983.

[33] R. King, A review of vortex shedding research and its application. Ocean Engineering 4(3) (1977) 141-171. doi:10.2514/3.5995

[34] T. Tamura, T. Miyagi, The effect of turbulence on aerodynamic forces on a square cylinder with various corner shapes. Journal of Wind Engineering and Industrial Aerodynamics 83(1) (1999) 135-145. doi:10.1016/S0167-6105(99)00067-7

[35] H. Fujita, The Characteristics of Aeolian Tone Generated from Elliptic and Rectangular Cylinders (in Japanese). JSME annual meeting 7 (2003) 257-258. 